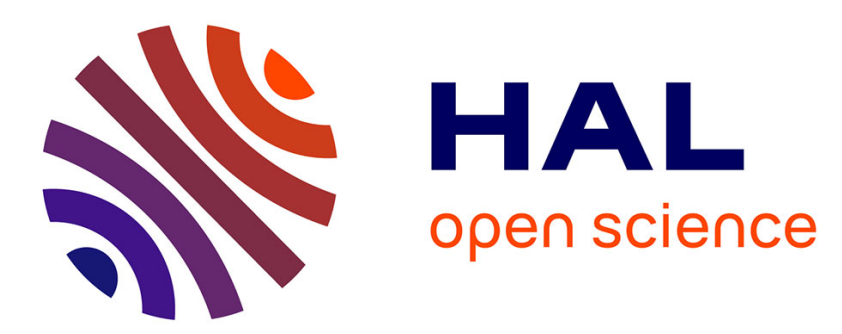

\title{
A Critical Comparison of Some Metrological Parameters Characterizing Local Digital Image Correlation and Grid Method
}

\author{
Michel Grédiac, Benoît Blaysat, Frédéric Sur
}

\section{- To cite this version:}

Michel Grédiac, Benoît Blaysat, Frédéric Sur. A Critical Comparison of Some Metrological Parameters Characterizing Local Digital Image Correlation and Grid Method. Experimental Mechanics, 2017, 57 (6), pp.871-903. 10.1007/s11340-017-0279-x . hal-01509611

\section{HAL Id: hal-01509611 \\ https://hal.science/hal-01509611}

Submitted on 11 May 2017

HAL is a multi-disciplinary open access archive for the deposit and dissemination of scientific research documents, whether they are published or not. The documents may come from teaching and research institutions in France or abroad, or from public or private research centers.
L'archive ouverte pluridisciplinaire HAL, est destinée au dépôt et à la diffusion de documents scientifiques de niveau recherche, publiés ou non, émanant des établissements d'enseignement et de recherche français ou étrangers, des laboratoires publics ou privés. 


\title{
A critical comparison of some metrological parameters characterizing local digital image correlation and grid method
}

\author{
Michel GrédiaC*, Benoît Blaysat*, Frédéric SuR ${ }^{\dagger}$
}

\begin{abstract}
The main metrological performance of two full-field measurement techniques, namely local digital image correlation (DIC) and grid method (GM), are compared in this paper. The fundamentals of these techniques are first briefly recalled. The formal link which exists between them is then given (the details of the calculation are in Appendix 1). Under mild assumptions, it is shown that GM theoretically gives the same result as DIC, since the formula providing the displacement with GM is the solution of the minimization of the cost function used in DIC in the particular case of a regular marking. In practice however, the way the solution is found being totally different from one technique to another, they feature different metrological performance. Some of the metrological characteristics of DIC and GM are studied in this paper. Since neither guideline nor precise standard is available to perform a fair comparison between them, a methodology must first be defined. It is proposed here to rely on three metrological parameters, namely the displacement resolution, the bias and the spatial resolution, to assess the metrological performance of each technique. These three parameters are thoroughly defined in the paper. Some of these quantities depend on external parameters such as the pattern of the surface of interest, so the same set of grid images is processed with both techniques. Only the contribution of the camera sensor noise to the displacement resolution is considered in this study. The displacement resolution, the bias and the spatial resolution are not independent but linked. These links are therefore studied in depth for DIC and GM and compared. In particular, it is shown that the product between the displacement resolution and the spatial resolution can be considered as a metric to perform this comparison. The extension to speckled patterns of the lessons drawn from grids is finally addressed in the last part of the paper. As a general conclusion, it can be said that for the value of the bias fixed in this study, the additional cost due to grid depositing offers GM to feature a better compromise than subset-based local DIC between displacement resolution and spatial resolution.
\end{abstract}

Keywords: digital image correlation, displacement, full-field measurement, grid method, metrology, strain.

This is the author-manuscript version of

M. Grédiac, B. Blaysat, F. Sur. A critical comparison of some metrological parameters characterizing local digital image correlation and grid method. Experimental Mechanics, SEM \& Springer, 2017.

DOI: $10.1007 / \mathrm{s} 11340-017-0279-\mathrm{x}$

*Université Clermont Auvergne, SIGMA, CNRS, Institut Pascal, BP 10448, 63000 Clermont-Ferrand, France

${ }^{\dagger}$ Université de Lorraine, LORIA, CNRS, INRIA projet Magrit, BP 239, 54506 Vandœuvre-lès-Nancy Cedex, France 


\section{Introduction}

There is a wide literature on the metrological performance of full-field measuring techniques used in experimental mechanics when they are considered individually. The influence of various parameters on systematic and random errors in the displacement and strain maps provided by such techniques seems to be the most widely addressed topic, especially concerning digital image correlation (DIC), see [1, 2, $3,4,5,6,7,8,9,10,11,12,13,14]$ for instance, or the grid method (GM) $[15,16,17,18]$. Comparing the performance of different techniques between each other (or even between various versions of the same technique) is, however, a major problem. Indeed, despite recent efforts [19, 20, 21, 22, 23, 24, 25], only a general standard is available [26], and no clear guide helping the user to perform such a comparison in an objective and systematic way is available on this subject. Comparing measuring techniques is however crucial to assist experimentalists intending to use the tool, which is the most appropriate to a given problem.

The main purpose of this study is to compare some parameters characterizing the metrological performance of two 2D white-light full-field measuring techniques: subset-based local DIC and GM. This problem is difficult to solve for two main reasons.

The first problem is to choose and define properly relevant parameters to characterize the metrological performance of full-field measurement systems. An international guiding document gathering the definitions of the main metrological parameters of any measurement technique is available, see Ref. [27]. We propose thus to rely on this document in the present study. The systematic error (or the bias) and the random error are considered here to characterize the metrological performance. The random error is assessed through its standard deviation which is also referred to as the measurement resolution. Since we deal here with full-field measurements, a third quantity must be considered in addition to the last two, namely the spatial resolution. The spatial resolution reflects the ability of a given technique to distinguish close features in a map. This last quantity is not defined in [27], so we will rely here on a recent definition proposed in the literature, as explained in detail in Section 5 below. Finally, we only focus here on the displacement, the strain being considered as a quantity deduced from the displacement by post-processing. Consequently, the measurement resolution is here the displacement resolution. An important feature is that the bias, the displacement resolution and the spatial resolution are linked. For instance, it is well-known that increasing the subset size in DIC causes the displacement resolution (thus the noise level) to decrease, but the counterpart is that the displacement distribution becomes more smoothed (thus blurred), which means that the spatial resolution is impaired and that the bias increases. Consequently, performing a fair comparison between two different techniques should consist in comparing more the functions linking these three parameters than comparing these parameters separately for given settings. Our methodology therefore relies heavily on predictive formulas available in the recent literature to compare the nature of this link between these parameters for each technique. These formulas being experimentally verified in other papers, they were considered as reliable to predict the metrological parameters.

Performing a fair comparison requires to address a second problem, the fact that the parameters defined above are influenced by various factors which are external to the measurement techniques themselves. Lighting spatial homogeneity or flickering, lens artifacts (vignetting or geometric distortion for instance), quality of the surface marking, micro-movement between specimen and camera which may occur while taking the pictures or out-of-plane displacements are typical examples of external factors influencing the results for a given technique or for a given version of a technique. This raises the question of the comparability of results obtained from images taken under different conditions, for instance at different places, or at the same place but at different times, thus under experimental conditions which are not strictly the same. This issue was tackled here by considering only the errors due to the image processing performed by 2D-DIC and GM, the impact of the external parameters described above affecting both techniques a priori in a similar way. The same set of patterns was also 
considered for both techniques when it was possible, or the results obtained with different patterns were normalized with respect to some relevant quantities which are detailed in the paper.

The paper is organized as follows. DIC and GM are briefly introduced in Section 2. The details of the formal link between these two techniques are given in Appendix 1. The three parameters considered here to compare the metrological performance of these two techniques are thoroughly defined in Section 2.3. The determination of these parameters is then examined for each technique. We will see that the bias has different causes which will be discussed separately. We compare finally the obtained values as well as the links betweens the three metrological parameters when they are available. The notations used in the paper are gathered in Table 1.

\section{A brief digest on DIC and GM and remarks on their metrological performance}

DIC and GM share a common feature: they are white-light techniques. However surface marking is generally different: GM relies on grids (but other periodic patterns may also potentially be employed), while random markings are usually employed with DIC. This is due to the very nature of these techniques since GM is a spectral approach while DIC works by comparing the gray level intensity between current and reference images. Note that the use of grids with DIC is reported to be successful in some papers, the natural contrast of the surface under investigation being enhanced, see [29] for instance. This remark will turn out to be important here, grid images being employed with DIC in several simulations performed to compare GM and DIC in the fairest way. Though Refs [30, 31] do not recommend the use of regular patterns, our own experience confirms that such markings work pretty well with DIC [29, 14]. The sole problem is the initialization of the iterative calculation used in DIC (see the following section), but it can be easily solved by using a pyramidal approach, as suggested in [32]. In conclusion, employing here the same type of marking for both DIC and GM enables us to fairly compare the core of these methods independently of the difference in marking that usual random patterns used in DIC would bring about. This approach is therefore used in the following sections. Speckle patterns used in DIC are however considered in the last section of the paper.

\subsection{Digital Image Correlation}

DIC is a full-field measurement method based, in its conventional form, on the minimization of a cost function with respect to the sought displacement. This cost-function reflects the difference between sub-images (or subsets) extracted from current and reference images. The procedure provides eventually the displacement at the center of each subset. There is a wide literature describing DIC and discussing the features of its main variants, see [31] for instance. More sophisticated approaches such as the global DIC (G-DIC), which is also referred to as finite-element based DIC, have been proposed in the literature (see [33] after [34]). Each approach has its own pros and cons [35, 36, 11,37]. However and for the sake of simplicity, we consider here the conventional version of DIC based on subset matching. The residual considered here is the sum of the squared difference (SSD) between the gray levels in subsets extracted in the current and reference images. An important point is that the minimization of the cost-function is iterative. This makes it difficult to elaborate closed-form expressions for various metrological parameters.

The principle of the local subset-based DIC is recalled here. Let $s^{r e f}(x, y)$ (resp. $\left.s^{\text {cur }}(x, y)\right)$ be the reference (resp. current) image. The cost function is the subset DIC residual $R_{\Omega}(\underline{u})$, which is the sum of the squared difference between the gray levels in subsets extracted in the current and reference images. In other words, for each subset $\Omega$ we have:

$$
R_{\Omega}(\underline{u})=\sum_{\left(x_{i}, y_{i}\right) \in \Omega}\left(s^{r e f}\left(x_{i}, y_{i}\right)-s^{c u r}\left(x_{i}+u_{x}\left(x_{i}, y_{i}\right), y_{i}+u_{y}\left(x_{i}, y_{i}\right)\right)\right)^{2} .
$$




\begin{tabular}{|c|c|}
\hline \multicolumn{2}{|c|}{ Notations for the camera and material data } \\
\hline$(x, y)$ (pixel) & Pixel coordinates in the (reference) camera sensor system \\
\hline$s^{r e f}$ & Reference image \\
\hline$s^{\text {cur }}$ & Current image \\
\hline$\underline{\tilde{u}}$ (pixel) & Reference (and sought) displacement occurring between the reference and the current configurations \\
\hline$f\left(\mathrm{pixel}^{-1}\right)$ & local reference displacement frequency \\
\hline \multicolumn{2}{|c|}{ Notations for the metrological quantities } \\
\hline$\underline{u}$ (pixel) & Displacement measurement of $\underline{\tilde{u}}$, see Equation 18 \\
\hline$b$ (pixel) & Bias \\
\hline$e$ (pixel) & Random error \\
\hline$d_{u}($ pixel $)$ & Spatial resolution for a given value of $\lambda_{2}$, see section 5.1 and 5.2 \\
\hline$d_{u}^{\prime} \quad($ pixel $)$ & Spatial resolution for a given value of $\lambda_{2}$ and $\lambda_{3}$, see section 5.4 \\
\hline \multirow[t]{2}{*}{$\alpha\left(\right.$ pixel $\left.^{2}\right)$} & metrological efficiency indicator for a given value of $\lambda_{2}$, \\
\hline & see Section 5.3 \\
\hline$\widetilde{\alpha}\left(\mathrm{pixel}^{2} / \mathrm{DN}\right)^{\dagger}$ & $\begin{array}{l}\text { normalized metrological efficiency indicator for a given value of } \lambda_{2} \text {, } \\
\text { see Section } 5.3\end{array}$ \\
\hline$\widetilde{\alpha}^{\prime}\left(\operatorname{pixel}^{2} / \mathrm{DN}\right)^{\dagger}$ & $\begin{array}{l}\text { normalized metrological efficiency indicator for a given value of } \lambda_{2} \text { and } \lambda_{3} \text {, } \\
\text { see Section } 5.4\end{array}$ \\
\hline$\widetilde{\widetilde{\alpha}}($ pixel) & $\begin{array}{l}\text { pattern-independent normalized metrological efficiency indicator for a given value } \\
\text { of } \lambda_{2} \text {, see Section 6.3.3 }\end{array}$ \\
\hline$\lambda_{1}($ pixel $)$ & Data interpolation bias, see section 3.1 \\
\hline$\lambda_{2}(-)$ & Attenuation bias, see section 3.2 \\
\hline$\lambda_{3}(-)$ & Displacement interpolation bias, see section 3.3 \\
\hline$\lambda_{4}(-)$ & Large-strain induced bias, see section 3.4 \\
\hline$\sigma_{u}$ (pixel) & Displacement resolution, see section 4 \\
\hline$\widetilde{\sigma}_{u}(\mathrm{pixel} / \mathrm{DN})$ & Normalized displacement resolution, see Equation 29 \\
\hline$\widetilde{\sigma}_{u}^{\prime}(\mathrm{pixel} / \mathrm{DN})$ & Normalized displacement resolution in the presence of Bias 3, see Section 5.4 \\
\hline$\widetilde{\sigma}_{u}(-)$ & Pattern-independent normalized displacement resolution, see Equation 40 \\
\hline \multicolumn{2}{|c|}{ Notations for DIC } \\
\hline$d(-)$ & degree of the matching function \\
\hline $2 M+1$ (pixel) & Subset size \\
\hline $\boldsymbol{L}_{\Omega}$ & Projection of DIC displacement shape function on the reference image gradient, see Equation 8 \\
\hline$M_{\Omega}$ & DIC tangent operator, see Equation 6 \\
\hline $\boldsymbol{P}_{\Omega}$ & Interpolation operator, once the DIC scheme has converged \\
\hline$\Omega(-)$ & subset \\
\hline \multicolumn{2}{|c|}{ Notations for GM } \\
\hline$p$ (pixel) & Grid pitch \\
\hline$\Phi(\mathrm{rad})$ & Phase modulation of the grid patterns \\
\hline$\Phi^{r e f}(\mathrm{rad})$ & Phase modulation of the reference image \\
\hline$\Phi^{\text {cur }}(\mathrm{rad})$ & Phase modulation of the current image \\
\hline$\sigma$ (pixel) & Standard deviation of the Gaussian window \\
\hline$a \quad$ (pixel) & Width of the triangular window \\
\hline
\end{tabular}

${ }^{\dagger}$ DN: Data Number, see [28]

Table 1: Main notations used in the paper.

While it is evaluated at the pixel coordinates $\left(x_{i}, y_{i}\right) \in \Omega$, an interpolation is required to elaborate the back-deformed image. It means that $s^{\text {cur }}$ in Equation 1 must be evaluated at points whose coordinates are those of the pixels in the reference coordinate system, to which the value of the sought displacement is added. The subsets are considered as squares, whose side is equal to $2 \times M+1$ pixels. In the examples discussed in this paper, $M$ is equal to $3,6,10,15$ or 20 pixels. The subsets are built up around their center. These subsets define a mesh. Within each subset, the displacement $\underline{u}$ is assumed to be of the first order, i.e. 
$\forall \Omega, \forall(x, y) \in \Omega, \exists\left(\mathbf{u}_{\Omega, k}\right)_{1 \leq k \leq 6} \in \mathbb{R}^{6}$ such that $\left\{\begin{array}{l}u_{x}\left(\boldsymbol{u}_{\Omega}\right)(x, y)=\mathbf{u}_{\Omega, 1}+\mathbf{u}_{\Omega, 3} \times x+\mathbf{u}_{\Omega, 5} \times y \\ u_{y}\left(\boldsymbol{u}_{\Omega}\right)(x, y)=\mathbf{u}_{\Omega, 2}+\mathbf{u}_{\Omega, 4} \times x+\mathbf{u}_{\Omega, 6} \times y\end{array}\right.$,

where $\boldsymbol{u}_{\Omega}$ is the $6 \times 1$ vector collecting the degrees of freedom $(\mathrm{dofs})\left(\mathbf{u}_{\Omega, k}\right)_{1 \leq k \leq 6}$. For the sake of simplicity, $x$ and $y$ are dimensions which are normalized with respect to the subset, thus $(x, y) \in$ $[-1,1]$. Consequently, $\mathbf{u}_{\Omega, 1}\left(\right.$ resp. $\left.\mathbf{u}_{\Omega, 2}\right)$ represents the $x$-component (resp. $y-$ ) of the subset center displacement.

For each subset $\Omega$, the best displacement field is considered as the one that minimizes the associated residual $R_{\Omega}$. The optimized dofs satisfy:

$$
\forall \Omega,\left(\mathrm{u}_{\Omega, k}\right)_{1 \leq k \leq 6}=\underset{\boldsymbol{u}_{\Omega}^{*} \in \mathbb{R}^{6}}{\operatorname{Argmin}}\left(R_{\Omega}\left(\underline{u}\left(\boldsymbol{u}_{\Omega}^{*}\right)\right)\right) .
$$

This minimization problem is rewritten as a root-finding one, such that for any $\Omega$, the six real numbers $\mathrm{u}_{\Omega, k}, 1 \leq k \leq 6$ satisfy the following equation:

$$
\forall k \in\{1, \ldots, 6\}, \frac{\partial R_{\Omega}\left(\underline{u}\left(\boldsymbol{u}_{\Omega}\right)\right)}{\partial \mathbf{u}_{\Omega, k}}=0 .
$$

A modified Gauss-Newton algorithm is implemented to solve this problem [38], and the iterative scheme is elaborated such that

$$
\forall \Omega, \boldsymbol{u}_{\Omega}^{\mathrm{it}+1}=\boldsymbol{u}_{\Omega}^{\mathrm{it}}+\boldsymbol{\delta} u_{\Omega}
$$

with $\delta \boldsymbol{u}_{\Omega}=\boldsymbol{M}_{\Omega}^{-1} \times \boldsymbol{b}_{\Omega}^{\text {it }}$ and $\boldsymbol{u}_{\Omega}^{0}=\mathbf{0} . \boldsymbol{M}_{\Omega}$ is a $6 \times 6$ matrix and $\boldsymbol{b}_{\Omega}^{\text {it }}$ a $6 \times 1$ vector. They are elaborated as follows, by using matrix $\boldsymbol{L}_{\Omega}$ and the residual $\boldsymbol{r}_{\Omega}\left(\underline{u}\left(\boldsymbol{u}_{\Omega}^{\text {it }}\right)\right)$ :

$$
\begin{aligned}
\boldsymbol{M}_{\Omega} & =\boldsymbol{L}_{\Omega} \times \boldsymbol{L}_{\Omega}^{t} \\
\boldsymbol{b}_{\Omega}^{\mathrm{it}} & =\boldsymbol{L}_{\Omega} \cdot \boldsymbol{r}_{\Omega}\left(\underline{u}\left(\boldsymbol{u}_{\Omega}^{\mathrm{it}}\right)\right) .
\end{aligned}
$$

Matrix $\boldsymbol{L}_{\Omega}$ and residual $\boldsymbol{r}_{\Omega}\left(\underline{u}\left(\boldsymbol{u}_{\Omega}^{\mathrm{it}}\right)\right)$ are defined such that

$$
\begin{aligned}
& \forall \Omega, \forall\left(x_{i}, y_{i}\right) \in \Omega, \forall k \in\{1, \ldots, 6\}, \\
& \qquad\left\{\begin{array}{l}
{\left[\boldsymbol{L}_{\Omega}\right]_{k, i}=\underline{\nabla}\left(s_{0}\right)\left(x_{i}, y_{i}\right) \cdot \frac{\partial}{\partial \mathbf{u}_{\Omega, k}} \underline{u}\left(\boldsymbol{u}_{\Omega}\right)\left(x_{i}, y_{i}\right)} \\
{\left[\boldsymbol{r}_{\Omega}\left(\underline{u}\left(\boldsymbol{u}_{\Omega}^{\mathrm{it}}\right)\right)\right]_{i}=s_{0}\left(x_{i}, y_{i}\right)-s_{1}\left(x_{i}+u_{x}\left(\boldsymbol{u}_{\Omega}^{\mathrm{it}}\right)\left(x_{i}, y_{i}\right), y_{i}+u_{y}\left(\boldsymbol{u}_{\Omega}^{\mathrm{it}}\right)\left(x_{i}, y_{i}\right)\right)}
\end{array} .\right.
\end{aligned}
$$

Since $\underline{u}\left(\boldsymbol{u}_{\Omega}\right)$ is of the first order, $\frac{\partial}{\partial u_{\Omega, k}} \underline{u}\left(\boldsymbol{u}_{\Omega}\right)$ does not depend on $\boldsymbol{u}_{\Omega}$ anymore. Hence matrix $\boldsymbol{L}_{\Omega}$ is constant as well as matrix $\boldsymbol{M}_{\Omega}$. Equation 5 is solved until the displacement increment $\left\|\boldsymbol{\delta} \boldsymbol{u}_{\Omega}\right\|$ is below some given threshold (set here to $10^{-5}$ ). The obtained displacement at the center of the subset is here directly defined thanks to the first two dofs.

$$
\underline{u}=\mathrm{u}_{\Omega, 1} \underline{e}_{x}+\mathrm{u}_{\Omega, 2} \underline{e}_{y}
$$

where $\left(\underline{e}_{x}, \underline{e}_{y}\right)$ is the orthonormal basis where the displacement is expressed. It is attached to the pixel directions of the camera sensor. The displacement map is obtained by interpolating the nodal displacements using the mesh associated with the subset centers. The interested reader is referred to Ref. [31] for more details.

\subsection{Grid Method}

GM only concerns regular patterns. We briefly present here how displacement fields can be deduced from a set of two grid images representing the current and reference configurations. 


\subsubsection{Grid model and phase modulation}

With the grid method, the sought displacement is contained in the phase change between current and reference grid images. A grid is indeed the superimposition of two perpendicular line patterns, each of them containing the information on one of the two components of the in-plane displacement, so two phase changes must be determined to retrieve the complete in-plane displacement.

In grid images, the light intensity $s(x, y)$ can be modelled as follows [39]

$$
s(x, y)=\frac{A}{2}\left(2+\gamma \cdot \operatorname{frng}\left(\frac{2 \pi x}{p}+\Phi_{x}(x, y)\right)+\gamma \cdot \operatorname{frng}\left(\frac{2 \pi y}{p}+\Phi_{y}(x, y)\right)\right)
$$

where:

- $A$ is the average global field illumination;

- $\gamma$ is the contrast of the oscillatory pattern between 0 and 1 ;

- frng is a real 0 -mean $2 \pi$-periodic function. Its amplitude is equal to 1 ;

- $p$ is the pitch of the two line patterns (the spatial frequency of the grid is therefore equal to $1 / p$ );

- $\Phi_{x}(x, y)$ and $\Phi_{y}(x, y)$ are the phase modulations of the line patterns along each direction.

Each in-plane displacement component $u_{i}, i \in\{x, y\}$ is proportional to the phase change, which is obtained by subtracting current and reference phase distributions. These quantities are denoted $\Phi_{i}^{c u r}$ and $\Phi_{i}^{r e f}, i \in\{x, y\}$, respectively. To obtain the displacement field, the first method is to subtract directly the current and the reference phase distributions, which gives

$$
u_{i}(x, y)=-\frac{p}{2 \pi}\left(\Phi_{i}^{\text {cur }}(x, y)-\Phi_{i}^{r e f}(x, y)\right) \quad i \in\{x, y\}
$$

The second approach consists in taking into account the movement of the physical points between current and reference images in the calculation of the displacement. Accounting for this movement is necessary to get rid of possible grid defects by subtracting their signature at the same physical points [17]. This gives

$$
u_{i}(x, y)=-\frac{p}{2 \pi}\left(\Phi_{i}^{c u r}\left(x+u_{x}(x, y), y+u_{y}(x, y)\right)-\Phi_{i}^{r e f}(x, y)\right) \quad i \in\{x, y\}
$$

In the second case, $u_{i}(x, y), i \in x, y$, is the solution of an implicit equation whose solution is found iteratively. Assuming that an initial estimation $u_{i}^{0}(x, y), i \in x, y$ is available (for instance with Equation 11$)$, it is possible to iteratively refine $u_{i}(x, y)$ through a fixed-point algorithm:

$$
u_{i}^{\mathrm{it}+1}(x, y)=-\frac{p}{2 \pi}\left(\Phi_{i}^{c u r}\left(x+u_{x}^{\mathrm{it}}(x, y), y+u_{y}^{\mathrm{it}}(x, y)\right)-\Phi_{i}^{r e f}(x, y)\right) \quad i \in\{x, y\}
$$

It can be checked with the fixed-point theorem that convergence is very rapid [17], one iteration being generally sufficient in the case of small strains. This point is discussed further in Section 3.4. With DIC, the solution is also found iteratively, but it relies on the Gauss-Newton algorithm, which is known to need more iterations to reach the same precision as the fixed-point algorithm, and each iteration involves a matrix inversion which is more demanding in terms of computational cost. Moreover, the numerical scheme used to minimize the residuals in Equation 3 may be trapped in a local minimum, while the solution of the fixed point problem in Equation 12 is unique. 


\subsubsection{Localized Spectrum Analysis}

Solving Equation 12 requires to estimate the phase modulations beforehand. Various Fourier-based image processing techniques can be used to process grid images to retrieve phase, displacement and strain maps, for instance sampling moiré [40], geometric phase analysis [41] and Windowed Fourier Transform (WFT). Concerning the WFT, two main approaches can be considered, either taking, in the Fourier domain, a set of frequencies close to the nominal grid frequency [42, 43], or picking only the nominal frequency of the grid and performing phase extraction with this sole frequency $[44,39,17]$. We propose to consider here only the last case for two main reasons (besides the computation time which is much shorter in this case):

- most of the recent examples available in the literature concerning real material characterization are based on this procedure;

- a big advantage is that closed-form expressions for the prediction of the metrological parameters are available in this case $[15,16]$, some of them having even been experimentally verified [16].

With this grid image processing technique named the Localized Spectrum Analysis [17], retrieving the phase from the signal $s$ consists of calculating the following complex number $\Psi(x, y, \theta)$ at any pixel $(x, y)$, where $\theta$ is the angle of the direction along which the information is obtained with the WFT.

$$
\Psi(x, y, \theta)=\int_{-\infty}^{+\infty} \int_{-\infty}^{+\infty} s(\xi, \eta) g(x-\xi, y-\eta) e^{-\frac{2 i \pi}{p}(\xi \cos (\theta)+\eta \sin (\theta))} \mathrm{d} \xi \mathrm{d} \eta
$$

where $g$ is a $2 \mathrm{D}$ window function. This function is symmetric, positive, and integrates to 1 . $\Psi(x, y, \theta)$ shall be calculated twice: with angle $\theta$ equal to 0 and $\frac{\pi}{2}$ to investigate the $x$ - and $y$-directions, respectively. Note that using an isotropic window enables us to employ grids rotated with respect to the $x-y$ axes of the camera sensor. This procedure has recently shown to be suitable to avoid aliasing in grid images, but is not detailed here (see [45] for more details). Various envelopes can potentially be employed in the WFT (see the discussion on the choice of the window in [18]). We consider two types of windows in this paper: the Gaussian and the bitriangular one. It can be shown that the former achieves the best compromise between various requirements, but its lower admissible width is such that its standard deviation $\sigma$ shall be greater than $p$, where $p$ is the pitch of the grid. This can be an issue if narrower windows must be employed to reveal localized features in displacement or strain maps. Hence we also consider the bitriangular window to complete the results obtained with the Gaussian window. Indeed the lowest admissible width $2 a$ is equal to $2 p$ for the bi-triangular window. This eventually leads to narrower windows compared to the Gaussian one, which can be an advantage in certain cases.

The function defining the Gaussian window is given by the following equation:

$$
g(x, y)=\frac{1}{2 \pi \sigma^{2}} e^{\left(-\frac{x^{2}+y^{2}}{2 \sigma^{2}}\right)}
$$

and the bitriangular one by:

$$
g(x, y)=\frac{a-|x|}{a^{2}} \times \frac{a-|y|}{a^{2}} \times \mathbb{1}_{[-a, a]}(x) \times \mathbb{1}_{[-a, a]}(y)
$$

where $\mathbb{1}_{[-a, a]}$ denotes the indicator function of interval $[-a, a]$. It is defined as $\mathbb{1}_{[-a, a]}(x)=1$ if $-a \leq x \leq a$ and $\mathbb{1}_{[-a, a]}(x)=0$ otherwise. The 3 -D graphical representation of $g$ is a square pyramid of height $\frac{1}{a^{2}}$, whose base is a square of dimension $[-a, a] \times[-a, a]$ in the $x-y$ plane. The conditions on the smallest admissible values for $a$ and $\sigma$ lead the smallest allowable window in the first case to be lower than in the second case, which is an advantage to detect localized features in a map. 
In the second case, however, $a$ shall be a multiple of $p$ to avoid potential parasitic fringes in the displacement/strain maps [18], while $\sigma$ can be equal to any real value greater than $p$ [15]. This gives more freedom when taking images of the grids and processing them to extract the sought information.

It is generally assumed that the argument of $\Psi(x, y, 0)$ and $\Psi\left(x, y, \frac{\pi}{2}\right)$ are equal to $\Phi_{x}(x, y)$ and $\Phi_{y}(x, y)$, respectively. A certain constant value $k$ must be added, but it has been demonstrated recently in [15] that this argument was, at first approximation and regardless of the noise, the phase of $s$ convolved by $g$ plus this constant value. Thus

$$
\operatorname{angle}\left(\Psi\left(x, y, \theta_{i}\right)\right)=g * \Phi_{i}+k, \quad i \in\{x, y\}
$$

where $k$ is a real number. In this equation, $\theta_{x}=0$ and $\theta_{y}=\frac{\pi}{2}$. This result will turn out to be useful to determine one of the metrological parameters of the technique, namely Bias 1 as defined in Section 2.3 below. $k$ disappears when performing the difference between current and reference phase distributions, so it is not detailed here. The argument of $\Psi(x, y, \theta)$ is calculated for each image, for each direction and at each pixel, which enables us to determine the two in-plane displacements using Equation 11 or 12, and then the strain components by differentiation. Note finally that Equation 17 holds modulo $2 \pi$, so the phase is discontinuous as soon as the amplitude of the phase change is greater than $2 \pi$ over a grid image, this jump being equal to $2 \pi$. A procedure named unwrapping shall thus be used to obtain a continuous distribution. This procedure is widely documented in the literature $([46,47]$ for instance) and various unwrapping programmes are available on the internet, so this point is not discussed further here. The programmes used in this study to process the grid images by GM are available in [48].

\subsection{General remarks on the metrological performances of DIC and GM}

It is shown in Appendix 1 that, under mild assumptions, the displacement found by the GM is theoretically the same as that found by DIC. The reason is that the difference in phase used in GM to obtain the displacement (see Equation 11) is the solution of the minimization problem addressed in DIC (see Equation 1), but in the particular case of a regular pattern such as a grid. In practice however, the way the solution is found is totally different from one technique to another. It means that if the same experimental conditions are used for both techniques (same lighting, gray depth, camera, ...), the difference between the performance of DIC and GM can only be due to various factors such as:

- the relevancy, for a given experiment, of the assumptions under which this calculation is performed;

- the stopping criterion fixed by the user in the iterative calculations performed for each technique;

- the method used to interpolate the information contained in the images;

- the sampling frequency;

- the way the noise propagates up to the final result for each technique;

- and the quality of the marking.

In this paper, we consider only the errors which are due to image processing to retrieve the displacement field from a set of reference and current images, and the objective is to characterize them.

As for the measurement of any physical quantity, the measurement denoted here $u_{i}, i \in\{x, y\}$ of the displacement components given by DIC and GM can be split into three different parts:

$$
u_{i}=\widetilde{u}_{i}+b_{i}+e_{i} \quad i \in\{x, y\}
$$

where 
- $\widetilde{u}_{i}$ is the reference (and sought) value;

- $b_{i}$ represents the bias as defined in $[27,49]$, i.e. the estimate of the systematic measurement error;

- $e_{i}$ represents the zero-mean random part of the error in the measurements.

With this notation, $b_{i}+e_{i}$ represents the measurement error for the displacement.

Any user of full-field measurement technique is seeking $\widetilde{u}_{i}$, which means that $b_{i}+e_{i}$ must be as small as possible. Both these quantities must be estimated to provide an uncertainty interval for the measurement of the displacement components. Comparing the metrological performance of two full-field measuring technique thus means that both quantities $\left(b_{i}\right.$ and $\left.e_{i}\right)$ must be estimated for each technique. The last one are zero-mean random values. The standard deviation characterizes the spread of these distributions. This standard deviation can be considered as the smallest reliable quantity that emerges from noise. It is named here the displacement resolution. An important point is that both the bias and the displacement resolution depend on the size of the region considered for calculating these quantities at a given pixel. This is logical because the bigger this zone, the greater the "averaging effect" over this zone, so the higher the impairment of the details in the displacement distribution and the lower the noise level. It means that a third parameter influences the bias and the displacement resolution: the so-called spatial resolution. It is a feature of full-field measurement systems, since measuring systems providing only point-wise quantities are not concerned. The three parameters presented above, namely the bias, the displacement resolution and the spatial resolution, are discussed in turn for both techniques in the following sections.

In this context, we propose here to focus our comparison on the three metrological parameters defined above, and on their links (if any) for a given technique. This latter approach was proposed recently for GM alone in $[16,17]$.

\section{Bias}

The bias at a given point of a displacement map is difficult to grasp because it is potentially the consequence of various phenomena. We propose here to consider the following causes, which are considered each as a different bias:

- Bias 1 , or bias due to the interpolation required to express the data describing the current state of deformation in the reference coordinate systems. This information is contained in the gray level images for DIC and the phase maps for GM;

- Bias 2, or attenuation bias;

- Bias 3, or displacement interpolation bias;

- Bias 4, or large-strain induced bias.

These phenomena will be quantified by a parameter denoted here $\lambda_{i}$, where $i$ is the number of the bias. As we shall see, $\lambda_{1}$ is not directly comparable with the others because it is a displacement. The others are relative losses of information due to the phenomena which are studied in turn.

\subsection{Bias 1, Data interpolation bias}

With both techniques, the images of the specimen which deforms are captured with a camera. The pixel grid of its sensor usually defines the reference coordinate system. Retrieving the displacements necessitates in both cases to map the data describing the current state of deformation in the reference 
coordinate system. The information which is mapped is however different from one technique to another. Let us examine below the impact of this mapping on the displacement field for each of the two techniques.

\subsubsection{Digital Image Correlation}

With DIC and using the procedure described in Section 2.1, the discretized images of the deformed patterns is back-deformed in the reference coordinate system to find the extremum of the correlation function (see Equation 1). The displacement being in general a non-integer value, interpolation between integer locations must be performed. This procedure induces a systematic error, which depends on various parameters, the main one being the subpixel displacement. The nature of the function used to perform this interpolation strongly influences the amplitude of this phenomenon. This amplitude is therefore difficult to predict and a wide literature is available on this subject, see $[50,51,52]$ for instance. Note that other strategies than back-deforming the current image in the reference coordinate system can be used while performing DIC (see the comprehensive review in [53]), but the presence of a systematic error is observed in all cases.

\subsubsection{Grid Method}

The procedure is different with GM since the images are first processed to extract the phase distribution of the reference and the current grid images, so it is not necessary to back-deform the current image. The current phase distributions can be directly subtracted with the reference phase distribution and the result multiplied by $\frac{-p}{2 \pi}$ (see Equation 11) to obtain a first estimate of the displacement. As explained above, this is however a "rough" estimate because subtraction is not performed at the same physical points, their movement during deformation being not taken into account. This induces potential perturbations which increase with the displacement. The safest route is therefore to map the current phase distribution in the reference coordinate system to be sure to subtract the information at the same physical point, which insures the impact of grid defects on displacement and mainly strain maps to be strongly attenuated, if not suppressed $[54,17]$. The procedure employed to map the phase distribution in the reference coordinate system also needs an interpolation between the integer locations of the pixels. The impact of these interpolations is studied below through a numerical simulation.

\subsubsection{Numerical simulation and comparison}

The consequence of the change of coordinate system presented above is illustrated with a simulation. This simulation consists of considering a 1000*1000-pixel image of a surface marked with a bidirectional grid created artificially with Equation 10. The pitch is equal to 5 pixels and the gray depth is 12 bits to mimic the response of a 12-bit camera. The theoretical profile of the grid lines is given by Equation 10, in which the frng function is a sine. The image is then shifted along the vertical $(y$-) axis, with a translation magnitude changing stepwise, the step being equal to $1 / 24$ pixel. At each step, the theoretical gray level distribution is merely obtained by adding a phase change of $\frac{-2 \pi}{24}$ to $y$ in Equation 10. The gray level at each pixel of the resulting images is found by integrating over each pixel the theoretical continuous gray level distribution given by Equation 10. A $3 \times 3$-point Gauss integration scheme is used for this purpose. This gives 25 images (the reference one and the 24 shifted ones) which are processed with the methodologies and settings discussed above. The first image of the series is considered as the reference image. The mean vertical displacement is then collected for each value of the subpixel displacement, and subtracted from the actual value which has been imposed to the image. A linear interpolation is deliberately used to interpolate the gray levels between integer values. Here, the number of pixels per grid period is equal to 5, which leads to local 
gray-level gradients which can be high. This is an unfavorable situation, which enhances the effect of Bias 1 for DIC, so the results presented here can be considered as an upper bound for DIC. There is a wide literature showing that better results are obtained with other types of interpolants [31, 51, 52], this bias being almost entirely cancelled out in certain cases. In Figure 1, we give for instance the result obtained with a cubic spline. It is clear that the bias is lower in this case than in the case of a linear interpolant. Employing more sophisticated interpolants would lead to even lower values of Bias 1, but would also induce additional computational expenses. This also certainly has a slight impact on the spatial resolution because interpolation relies on information at pixels surrounding that for which interpolation is performed. This point is not discussed in the literature, to the best of the authors' knowledge, and is therefore out of the scope of this paper. The subset size considered to plot the curves in Figure 1 is $2 M+1=21$ pixels, but other sizes give similar results. This gives rise to the classic "S-shaped" curve shown in Figure 1. With a linear interpolant, it is null for integer values of the displacement, as well as for a subpixel displacement equal to 0.5 for symmetry reasons. In the present calculation, this bias is maximum when the subpixel displacement is equal to 0.25 and 0.75 pixel. These locations change in the presence of noise, as discussed in [55].

The same set of images is processed with GM. A Gaussian window with $\sigma=5$ pixels is used in this case. A linear interpolation is also used for GM, but this time on the current phase distribution, not on the gray levels between adjacent pixels of the images. The value for the displacement returned by the grid image processing is averaged over the displacement map and subtracted to the reference one which was imposed in the grid images. The residual is considered as due to the interpolation. The resulting curve is superimposed to that obtained with DIC in Figure 1. The main conclusion is that the response is notably different between DIC and GM since only DIC is prone to a bias which is observable in the figure. There are two main reasons for this:

- the first reason is that the nature of the data which are interpolated are different from one technique to each other. We deal with integer values (the gray level of the images) with DIC, and floating numbers with GM (namely the phases, which are also estimated from the integer gray values). Indeed, interpolating integer values with DIC a priori leads to larger errors than interpolating real values with GM.

- the second reason is that the quantity to be interpolated exhibits gradient amplitudes which are different from one situation to another: the gray level must feature high gradients to ensure a good contrast, while the phase distribution generally gently evolves over the deformed surface, apart from the case of singularities like those occurring near crack tips for instance. This leads the resulting displacement field to be nearly not impacted by interpolation.

\subsection{Bias 2, or attenuation bias}

This bias comes from a rather intuitive phenomenon: both techniques provide information at isolated pixels, but this information depends on the gray level at the surrounding ones. Hence some kind of spatial averaging effect occurs. For instance, considering a displacement simulated by a sine function, the amplitude of the displacement returned by DIC and GM is all the more affected by image processing as the frequency of the sought distribution is high. This impairment is also all the greater as the size of the region considered by the user for calculating the displacement is large, this calculation acting as a low-pass filter, whose size is inversely proportional to the size of the region.

\subsubsection{Digital Image Correlation}

Concerning DIC, this bias comes from the fact that the displacement within each subset is modeled by a polynomial shape function. This function is supposed to mimic and match the actual displacement 


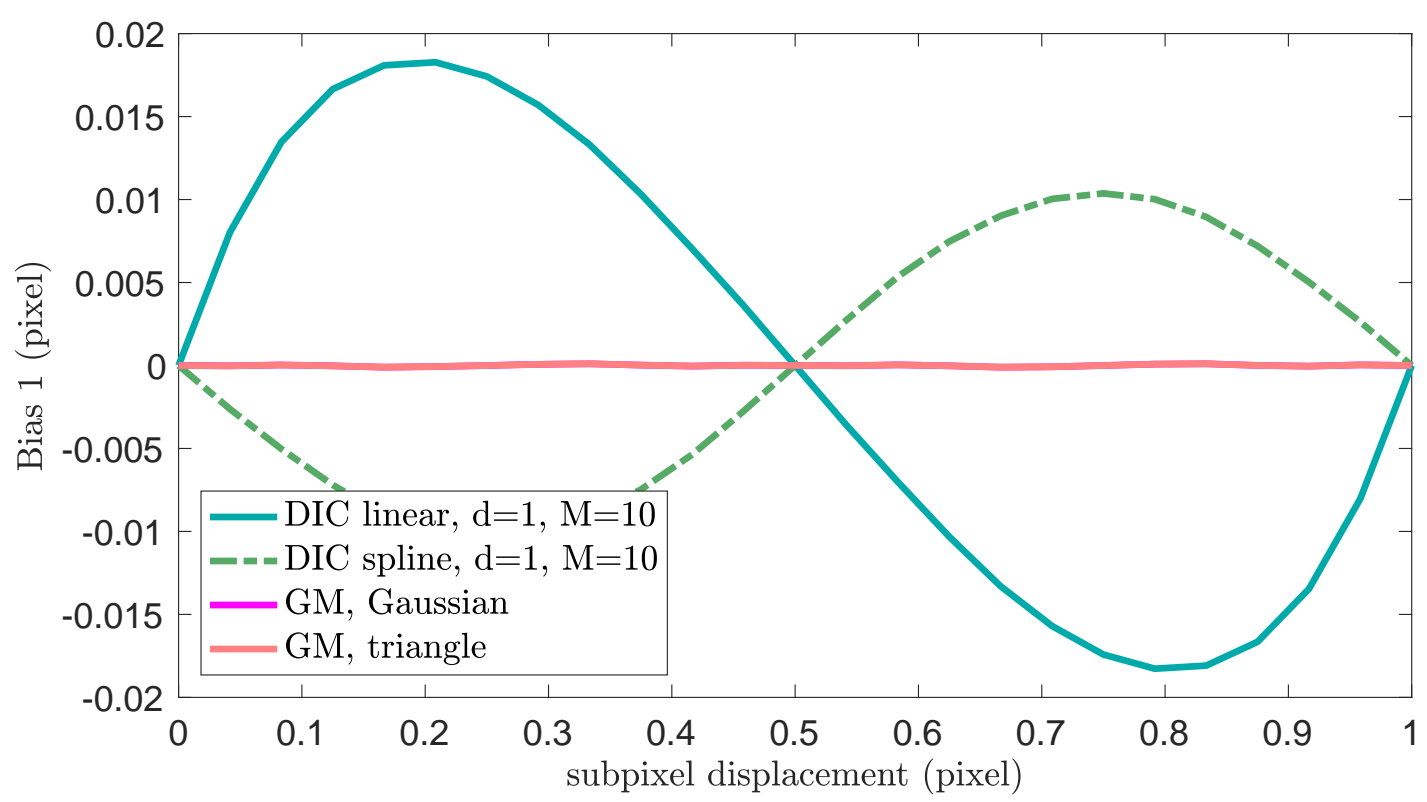

Figure 1: Bias 1 vs. subpixel displacement. Both curves for GM are superimposed.

within each subset, but it is clear that the quality of the matching depends on this function and on the real displacement distribution.

It is shown in [30] that the displacement at the center of the subsets returned by DIC can be considered as the actual one filtered by a Savitzky-Golay (SG) low-pass filter. This result is obtained if polynomial shape functions (characterized by their degree $d$ ) are employed to represent the displacement of the subsets between reference and current images. This is due to the fact that a local least-square fitting can be presented as a convolution by a kernel, which does not depend on the nature of the curve which is fitted [56]. It is worth noting that this conclusion does not take into account other sources of disturbance such as sensor noise propagation (this issue is discussed in Section 4) or imprecision inherent to the numerical scheme, DIC relying on iterative calculations.

\subsubsection{Grid Method}

A similar result as the preceding one has been demonstrated in [15] for GM: the phases (and thus the displacement and the strain components) retrieved by the WFT are equal, at first approximation and regardless of noise, to their actual and sought counterparts convolved by the analysis window employed in the WFT [15]. This is a consequence of Equations 11 to 13, and 17.

\subsubsection{Theoretical analysis}

Even though no matching function is employed with GM, we have here the same property for both techniques: they return quantities which are not exactly the actual ones, regardless of noise, interpolation, and other sources of errors like those discussed in the other sections. The amplitude of Bias 2 is therefore directly comparable between DIC and GM, Bias 2 being merely due to a convolution of the actual and sought displacement distribution by a kernel known a priori for each technique. In practice, Bias 2 is defined as the relative loss of amplitude $\lambda_{2}$ of a given sine displacement distribution. It can be directly assessed for each technique from the transfer function $\widehat{h}$ of its corresponding filter characterized by its impulse response $h$, namely the SG filter for DIC and the Gaussian or the trian- 
gular filter for GM. This transfer function is the Fourier transform of the filter $h$. Indeed, in signal processing, the transfer function of any linear filter is defined as the Fourier transform of its impulse response. Thus

$$
\lambda_{2}=1-\widehat{h}
$$

Applying such a filter to a reference sine signal of frequency $f$ provides a sine function featuring the same frequency. Its amplitude is, however, affected since it is equal to the amplitude of the sought signal multiplied by the value of the modulus of the transfer function for frequency $f$, the complex phase being shifted when the transfer function is not a real function. In the present situation, however, it should be noted that $\widehat{h}$ is real and symmetric with respect to 0 , as $h$. Consequently, the relative loss of amplitude $\lambda_{2}$ is always a real number.

Without loss of generality, we reduce here the discussion to the 1D-case for the sake of simplicity. The extension to the $2 \mathrm{D}$ case is straightforward because the filters used in the 2D-case are separable with respect to each direction. In this $1 \mathrm{D}$ case, the transfer function $\widehat{h}$ of a filter $h$ is generally given as a function of the spatial frequency $f$ of the signal in the continuous case. If the filter support is $\{-M,-M+1, \ldots, M-1, M\}$ (i.e., it is null outside these $2 M+1$ points), we shall consider the transfer function of the discrete filter denoted here $\widehat{h}_{M} . \widehat{h}_{M}$ is usually expressed as a function of the normalized wave number denoted $\widetilde{k}$, as in the seminal paper [30]. This dimensionless quantity is defined as the frequency $f$ divided by the Nyquist frequency, which is equal here to $\frac{1}{2} \operatorname{pixel}^{-1} \cdot \lambda_{2}(\tilde{k})$ is therefore given by:

$$
\lambda_{2}(\widetilde{k})=1-\widehat{h}_{M}(\widetilde{k})=1-\left(h(0)+2 \sum_{i=1}^{M} h(i) \cos (i \widetilde{k} \pi)\right)
$$

where $\tilde{k}$ lies between 0 and 1 . This equation holds thanks to the symmetry of $h$. With this notation, the discrete Fourier coefficients of $h$ are given by $\widehat{h}_{M}(\tilde{k})$ where $\tilde{k}=2 k / N$ and $N$ is the number of samples of the input signal, $k$ being any integer between $-N / 2$ and $N / 2-1$ (for even values of $N$ ). As mentioned earlier, $\widehat{h}_{M}$ is, as $h$, symmetric with respect to 0 . In order to make comparisons between the relative loss of amplitude independent from $N$, we adopt the same presentation as in Ref. [30], and plot graphs of $\lambda_{2}(\widetilde{k})$ against real values of $\widetilde{k}$ spanning the $[0,1]$ interval.

For DIC, the transfer function of a SG filter of degree $d$, discretized over $2 M+1$ points, is denoted here $\widehat{h}_{M, d}^{D I C}$. The values of the corresponding SG coefficients $h_{M, d}^{D I C}(i)$ are defined and tabulated in [56]. If $d=0$, the SG filter is a box filter of width $2 M+1$, hence $h_{M, 0}^{D I C}(i)=\frac{1}{2 M+1} \forall i \in[0, M]$. The value returned by the SG filter is therefore the mean value of the displacement over the subset but no strain can be determined [30]. A first-degree SG filter accounts for straining. For symmetry reasons, the displacement returned at the center of the subset is the same as that returned by a zerodegree SG filter $[30,56]$. Increasing this degree provides then more freedom to describe the actual displacement fields, so we considered $d=1,2$ in this section. We chose $M=3,6,10$ pixels only and not $M=15,20$ pixels, the $h_{M, d}^{D I C}(i)$ coefficients being not available in [56] for these last two values in the case $d=2$. The value of the coefficients employed here is recalled in Appendix 2, see Tables 3 to 5 .

For GM, the situation is slightly different because the calculation of the Fourier transform of classic windows employed in the WFT is tractable, which then enables us to analyze their properties more easily, as in [15] and [18] for instance. For the two types of windows considered here, we have therefore a transfer function which is given directly as a function of the frequency $f$ of the signal 


$$
\left\{\begin{array}{l}
\widehat{h}^{G M-G}(f)=e^{-2 \pi^{2} \sigma^{2} f^{2}} \text { for a Gaussian window of standard deviation } \sigma \\
\widehat{h}^{G M-T}(f)=\operatorname{sinc}^{2}(a f) \text { for a triangular window of width } 2 a
\end{array}\right.
$$

"sinc" denotes here the cardinal sine function defined by $\operatorname{sinc}(x)=\frac{\sin (\pi x)}{\pi x}$ for $x \neq 0$ and $\operatorname{sinc}(0)=1$.

To make Bias 2 calculated for DIC and GM comparable, it is, however, necessary to consider the discrete transfer function over $2 M+1$ points of the windows used in the WFT, not its continuous versions given in Equation 21. In the same spirit as for the SG filter, these functions are denoted $\widehat{h}_{M}^{G M-G}$ for a Gaussian window and $\widehat{h}_{M}^{G M-T}$ for a triangular one. Bias 2 is deduced by considering again Equation 20, but the $h(i)$ coefficients, $i=1 \cdots M$, are now the values of either the Gaussian or the triangular windows at the $2 M+1$ points where the signal is sampled.

Using this approach, Figure 2 shows Bias 2 for DIC and GM. Bias 2 is deduced from the transfer function of the SG filter function for various values of $M$ for DIC, and from the transfer function of the Gaussian and triangular filters for GM.

Several remarks can be drawn from these curves:

- Bias 2 is null for $\widetilde{k}=0$. Indeed, $\widehat{h}_{M}(0)=1$ since it is the mean value of the filter $h_{M}$, which is allways equal to 1 in order that it does not change constant signals. Consequently both DIC and GM return an unbiased value for a null wave number, which corresponds to a flat displacement distribution. The information becomes, however, biased for both techniques as soon as the frequency of the displacement becomes different from zero;

- concerning the size of the zone over which the displacement is retrieved, GM shares the same trend as DIC: the narrower this zone (thus the narrower the subset for DIC and the narrower the analysis window for GM), the better the ability to return a value for the displacement close to the actual one, and thus the lower the bias. Note that the Gaussian curve has not a compact support, but its "apparent width" can be estimated to $6 \sigma$ according to the classic $3-\sigma$ rule [57]. Bearing in mind that the analysis windows employed here are the narrowest that can be used for GM, it is worth mentioning that DIC is able to provide a displacement at a given pixel by relying on a smaller zone than the minimum one required for GM;

- for DIC and for a given value of $d$, all the curves all the more sharply increase as the subset is wide, which illustrates an averaging effect which becomes more pronounced when the subset is wide;

- for DIC and as already mentioned in [31], the benefit of a second-degree polynomial matching function clearly appears by comparing Figures 2-a and 2-b, representative curves of Bias 2 being shifted to the right from the first to the second degree whatever the size of the subset. This is a consequence of the fact that the shape function is a polynomial of greater degree, and has therefore "more freedom" to describe the actual displacement field. Interestingly, the representative curves for DIC are globally on the right of the curve representing the response of GM with a Gaussian filter and $\sigma=5$ pixels. The case defined by DIC, $d=2$ and $M=3$ is the best case, but its practical implementation seems to be difficult, the number of pixels covered by this subsets being limited compared to the number of unknowns to be fixed in the matching function;

- the curves for DIC exhibit oscillations and become even greater than one for some frequencies, which means that the signal returned by DIC at the centers of the subsets may not only be attenuated, but affected by a wrong sign for these frequencies. This feature is illustrated in the following section with a example. 

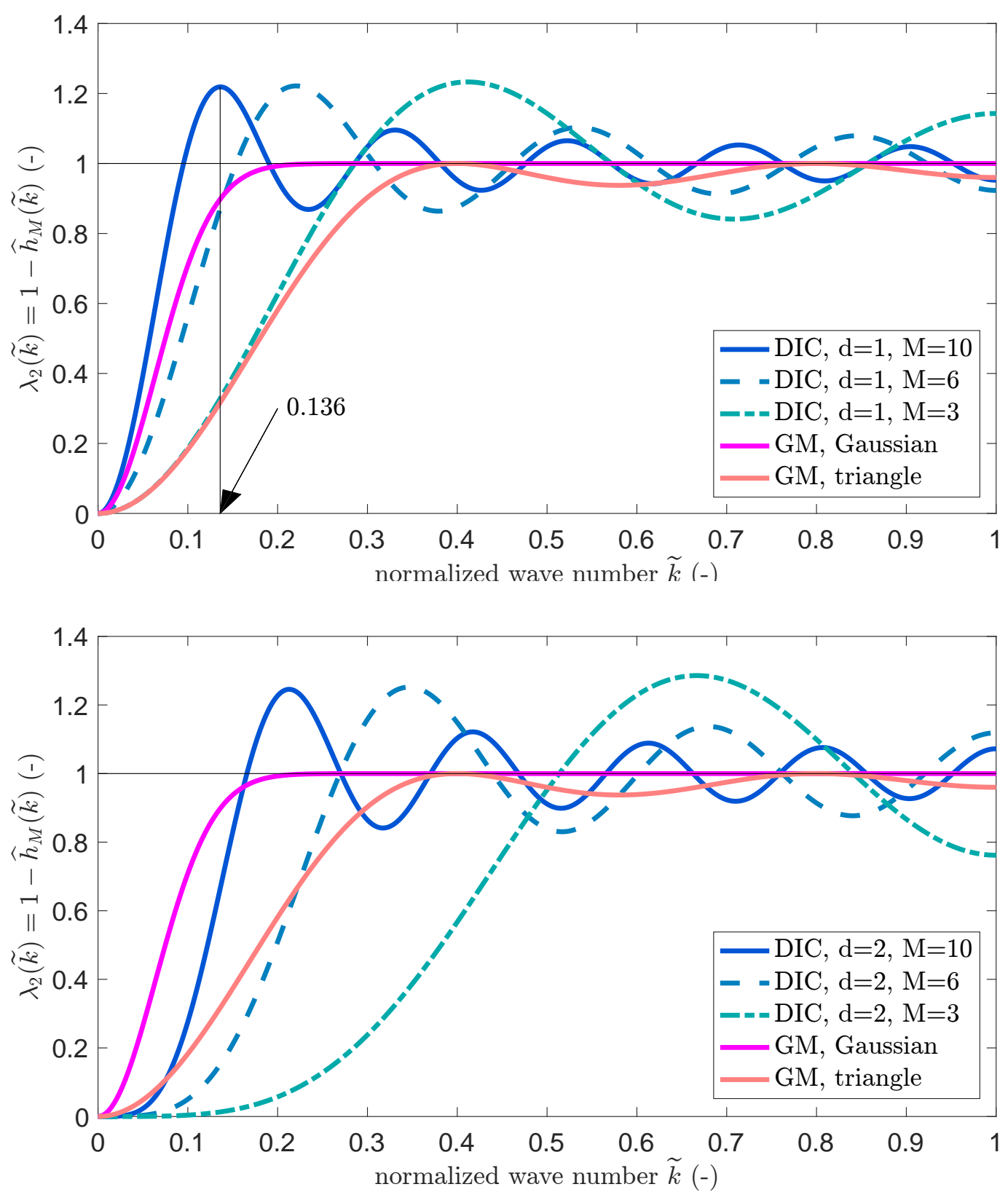

Figure 2: Comparison between Bias 2 obtained by DIC with linear (top) and quadratic (bottom) matching functions, $M=3,6,10$ pixels (shades of blue). The values for Bias 2 obtained by GM with a Gaussian window in the cases $\sigma=5$ pixels and a triangular window in the case $a=5$ pixels are superimposed (shades of red). 
- Bias 2 for GM in the case of the Gaussian and the triangular windows remains lower than (or equal to) one, so no sign error occurs with GM whatever the value of the frequency;

- slight oscillations can be observed for the representative curve of Bias 2 for a triangular window. They are due to the sinc ${ }^{2}$ function involved in the continuous version of Fourier transform of the filter (see Equation 21-b). Their amplitude is however smaller than that observed with DIC;

- the Fourier transform of a Gaussian function being also a Gaussian function, we have the same remark for the continuous version of the transfer function of the Gaussian function. As a consequence, Bias 2 for GM in the case of a Gaussian window does not theoretically exhibit any oscillation, which is the most favorable case. No oscillation is observed neither to the naked eye in the discrete version used here (a sum of cosine functions, see Equation 20). It means that high frequencies vanish in the displacement fields retrieved by GM. This causes the maps to be only slightly blurred. The price to pay to avoid these oscillations is, however, that the minimum size of the standard deviation of the Gaussian envelope is greater than the minimum size of the subset which can be used in DIC in practice.

\subsubsection{Numerical simulation}

An exemple is proposed here to illustrate the effect of Bias 2. It consists of a synthetic vertical displacement map which features an a priori known and spatially changing frequency in the same spirit as in $[2,11]$ for instance for DIC, and later in $[58,17]$ for GM. Figure 3-a shows this reference displacement field. It is such that the period of the displacement along the $y$-direction linearly evolves along the $x$-direction: from 10 to 150 pixels. The minimum period (10 pixels) corresponds to twice the typical smallest number of pixels generally considered to discretize grids, namely 5 pixels. The amplitude of the reference displacement wave is constant and equal to 0.5 pixel, which corresponds to the most favorable case for Bias 1 in DIC, as discussed in the preceding section. Hence this choice allows to emphasize the effect of Bias 2 for DIC. In the same spirit, no interpolation between remote points is performed here in order to get rid of the influence of Bias 3 discussed later in Section 3.3. For this purpose, the DIC subsets are defined with a shift of only 1 pixel, which is not a classic setting in DIC because of the demanding computation time. The DIC output displacement field is thus directly a full displacement field defined pixelwise and exhibiting the same size as that of the image. There is no extra interpolation step. It is worth mentioning that no particular precaution has been made for GM; the default option is generally to perform the calculation pixelwise because it is very fast.

This reference distribution is then filtered with the different filters discussed above (SG for DIC, Gaussian and triangle for GM). The change in period of the reference wave along the horizontal direction $(x)$ is negligible at the scale of the subset in DIC or the window in GM. Hence we can reasonably consider this problem as a succession of $1 \mathrm{D}$ problems defined along the $y$-direction, which means that the 1D filters discussed above can be applied along the $y$-direction for each column.

Figure 3-b shows an example of displacement field. A SG filter, with $M=10$ pixels and $d=1$, is employed here. The filtered signal is significantly attenuated on the left-hand side. It is null for a certain value of the period, and its sign even changes over a certain interval of periods as discussed above when $\lambda_{2}>1$. The attenuation of the amplitude clearly decreases when considering increasing periods for the reference displacement wave, thus when going to the right in Figure 3.

Figure 3-c shows the displacement field really obtained by DIC. The image processed here is that of a grid image modulated by the displacement imposed in Figure 3-a, and DIC is applied pixelwise, without interpolation (thus Bias 3 is null). No real difference can be observed to the naked eye between Figure 3-b and Figure 3-c (apart from the border effect which have been removed in Figure 3-c), which means that the SG filter correctly models the real response of DIC in terms of Bias 2 in this case.

We consider now in Figure 4 the distribution of Bias 2 along a cross-section of this displacement field. This cross-section is the horizontal symmetry axis. It is represented by a red line in Figure 3 . 

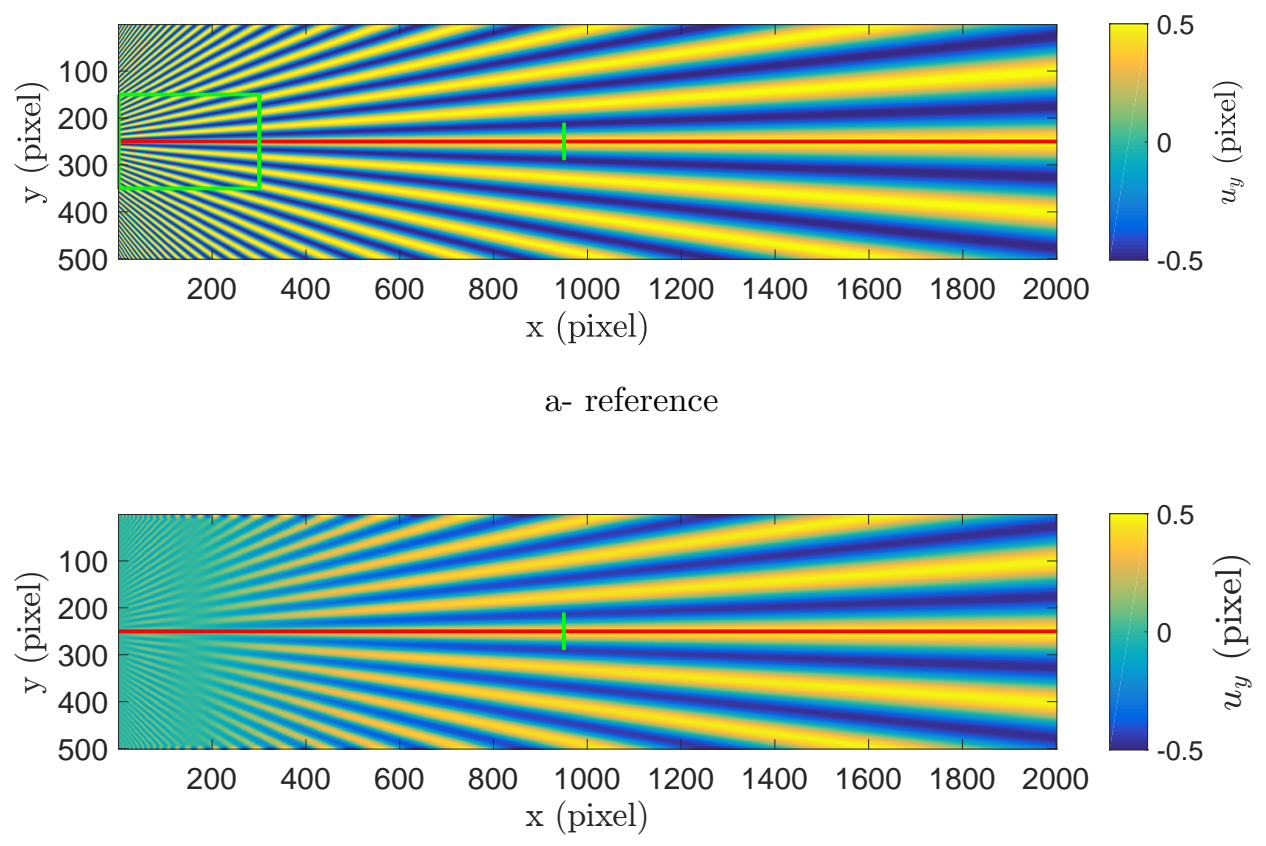

b- obtained by filtering the reference displacement field above with a SG filter

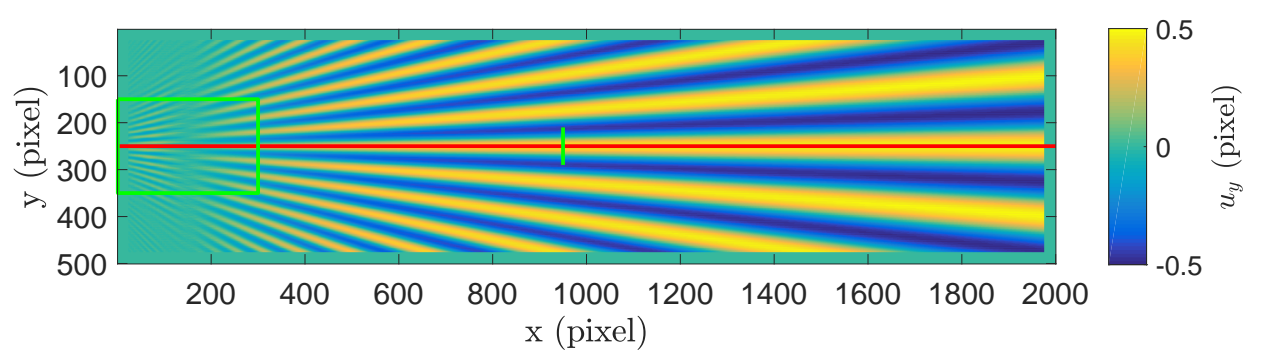

c- really obtained by DIC, no interpolation between remote points (shift=1).

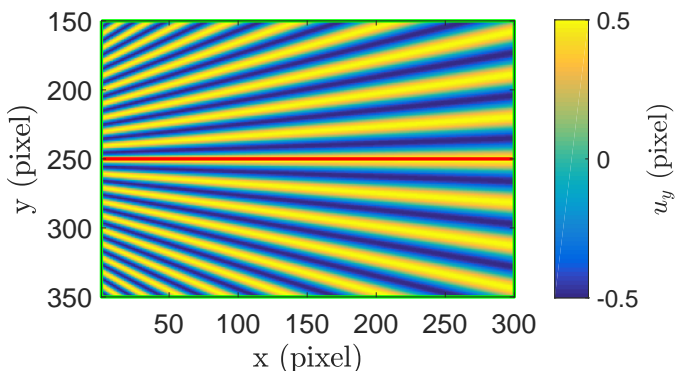

d- close-up view of the reference distribution

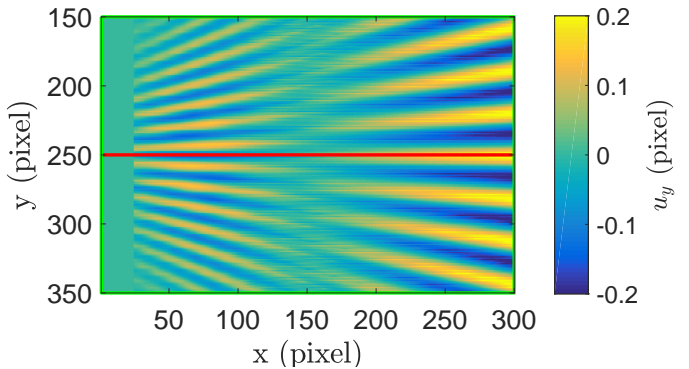

e- close-up view of the distribution retrieved by DIC (colorbar different in d- and e-)

Figure 3: a- Reference displacement map $u_{y}$. b- Example of displacement map filtered by a SG filter, $M=10$ pixels, $d=1$. c- Example of displacement map retrieved pixelwise with DIC, $M=10$ pixels, $d=1$. d- close-up view of the reference distribution. The corresponding zone is bounded by the green rectangle in sub-figure a- and c-. e- close-up view of the distribution retrieved with DIC. The sign flip is clearly visible with the interweaving of the blue and yellow waves between each side of the vertical line $x \simeq 150$ pixels. The location of this line moves rightwards when $M$ increases. The range of the colorbar is different in sub-figures d- and e- to highlight the phenomenon. 
A first order-SG filter (resp. second order) is employed to plot Figure 4-b (resp. Figure 4-c). The full lines correspond to the bias calculated with Equation 19, but represented as a function of the period of the sinusoidal displacement (it is equal to $\frac{1}{f}=2 \times 5 / \widetilde{k}$ ) instead of $\widetilde{k}$ directly. DIC and GM are considered with $M=3,5$ and 10 pixels and first-degree SG filters in Figure 4-a, and second-degree SG filters in Figure 4-b. The curves representative of GM are obtained for the sharpest analysis windows which can be used with this technique, namely a Gaussian window with $\sigma=p=5$ pixels and a triangular window with $a=5$ pixels. The consistency between the results in Figures 2 and 4 can be easily checked. A closeup view of Figure 2-a is shown in Figure 4-a for this purpose. As an example, the minimum value of the transfer function of the first-degree SG filter with $M=10$ pixels is obtained for a normalized wave number approximately equal to 0.136 (see Figure 4 -a). This corresponds to a period equal to $1 / 0.136 / 0.5 \simeq 14.7$ pixels in Figure 4-b.

The main remark that can be drawn from Figure 4 is that the highest biases are globally obtained for the smallest pitches of the prescribed sinusoidal displacement, the presence of oscillations for the SG filters changing the precise location of the worst case from one value of $M$ to another. The bias becomes even greater than one in some cases, which is the consequence of a change of sign in the signal after filtering, as can be seen on the left-hand side of Figures 3-b and 3-c. For DIC, this phenomenon becomes more pronounced when using wider subsets (not represented here). This is a consequence of the fact that the shape functions becomes progressively unable to reliably describe the real displacement [30]. This remark underlines that the subset size and the degree of the matching function must be carefully chosen to avoid obtaining wrong results. When possible, these settings must be such that the right-hand part of the curves in Figure 2 and the left-hand part of the curves in Figure 4-b and -c are not activated. Another remark is that as expected, second-degree shape functions provide better results than first-degree ones. The problem is that to the best of the authors' knowledge, most of the commercial DIC packages rely on first-degree polynomial matching functions, probably to limit the computational cost. Consequently their users cannot increase this degree if they wish to do so. In addition, overmatching may induce additional errors [59], and we observed during the present study that the case $M=3$ could only work with $d=1$, the case $d=2$ leading to unexpected results, probably because the information contained in the subset was too poor in comparison to the freedom offered by a second-degree matching function.

The dashed lines in Figure 4-a represent Bias 2 which is really obtained by DIC and GM. The images processed here are those of a grid image modulated by the displacement imposed in Figure 3a, and DIC is applied pixelwise, without interpolation (thus Bias 3 is null). There is a very good agreement between results obtained with Equation 19 and those obtained by applying really DIC and GM, which lends credence to the theoretical predictions on which the results given in this section rely. As a last remark, it must be emphasized that any real reference displacement or strain field can be expressed in Fourier series, after taking the discrete Fourier transform of the corresponding maps. Consequently, the issues with high frequencies discussed in this section not only concern the theoretical displacement field shown in Figure 3-a, but any field, the terms of the Fourier series being all the more affected as the frequency increases.

We consider only the case $d=1$ in the remainder of the paper for the sake of simplicity. In addition, most of the commercial DIC codes feature bilinear matching functions.

\subsection{Bias 3, or displacement interpolation bias}

With the subset-based DIC, displacements collected at the subset centers must be interpolated to deduce the displacement at other places located between the centers of the subsets in order to obtain a full-field map. This is generally done by interpolating the displacements at the nodes where this information is provided by DIC, or smoothing them for further calculation of the strain components. This eventually gives an interpolation error, which is all the higher as the strain gradient is high, and the distance between interpolated points high. In addition, Bias 2 described above propagates 


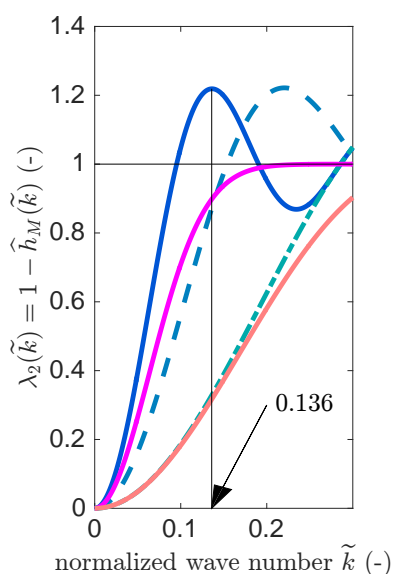

a- close-up view of Figure 2

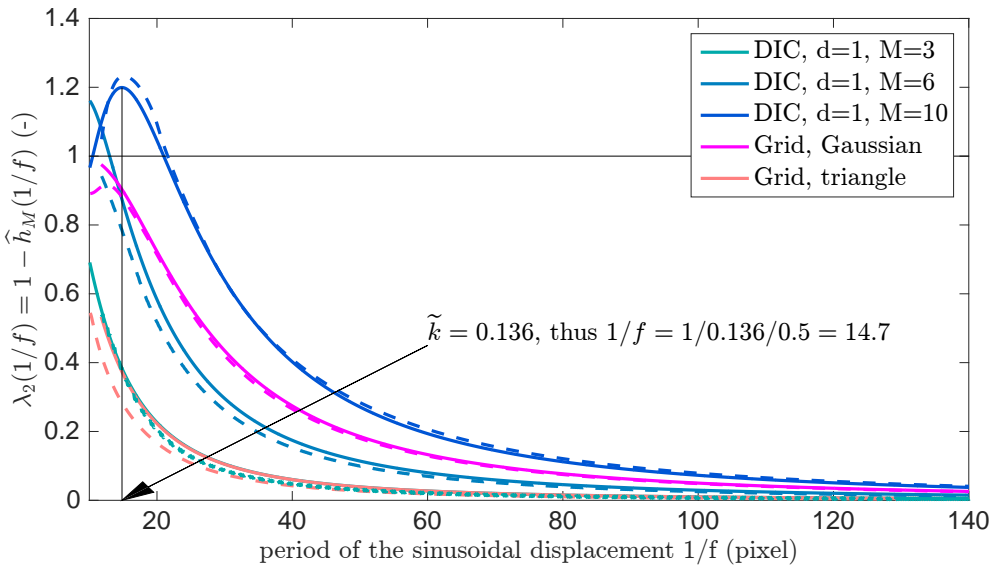

b- zero/first-degree SG filter

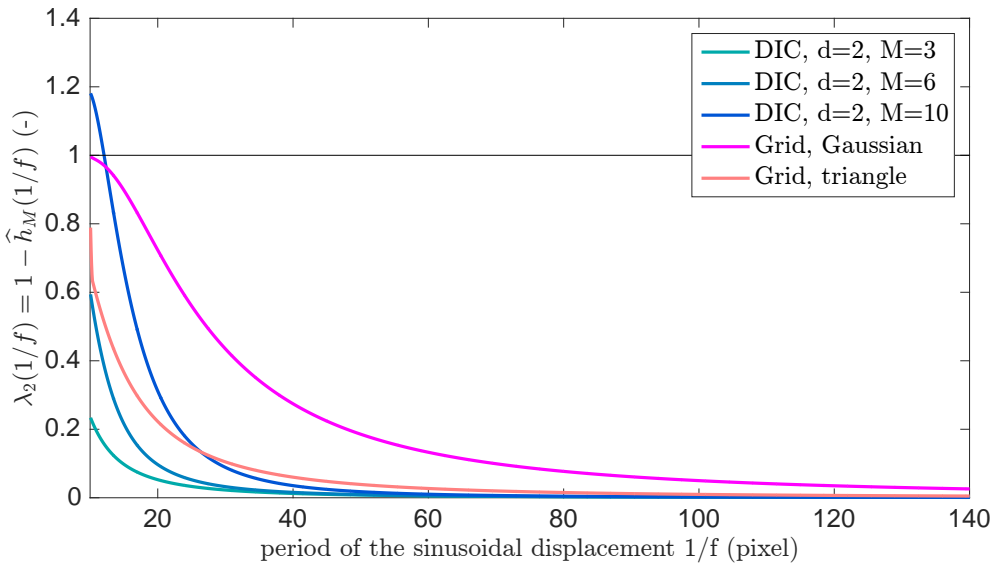

c- second-degree SG filter

Figure 4: Bias 2 for the zero/first-degree (top) SG filter, the second-degree (bottom) SG filter, the Gaussian and the triangle filters (the dashed line represent the result really obtained after running DIC). 


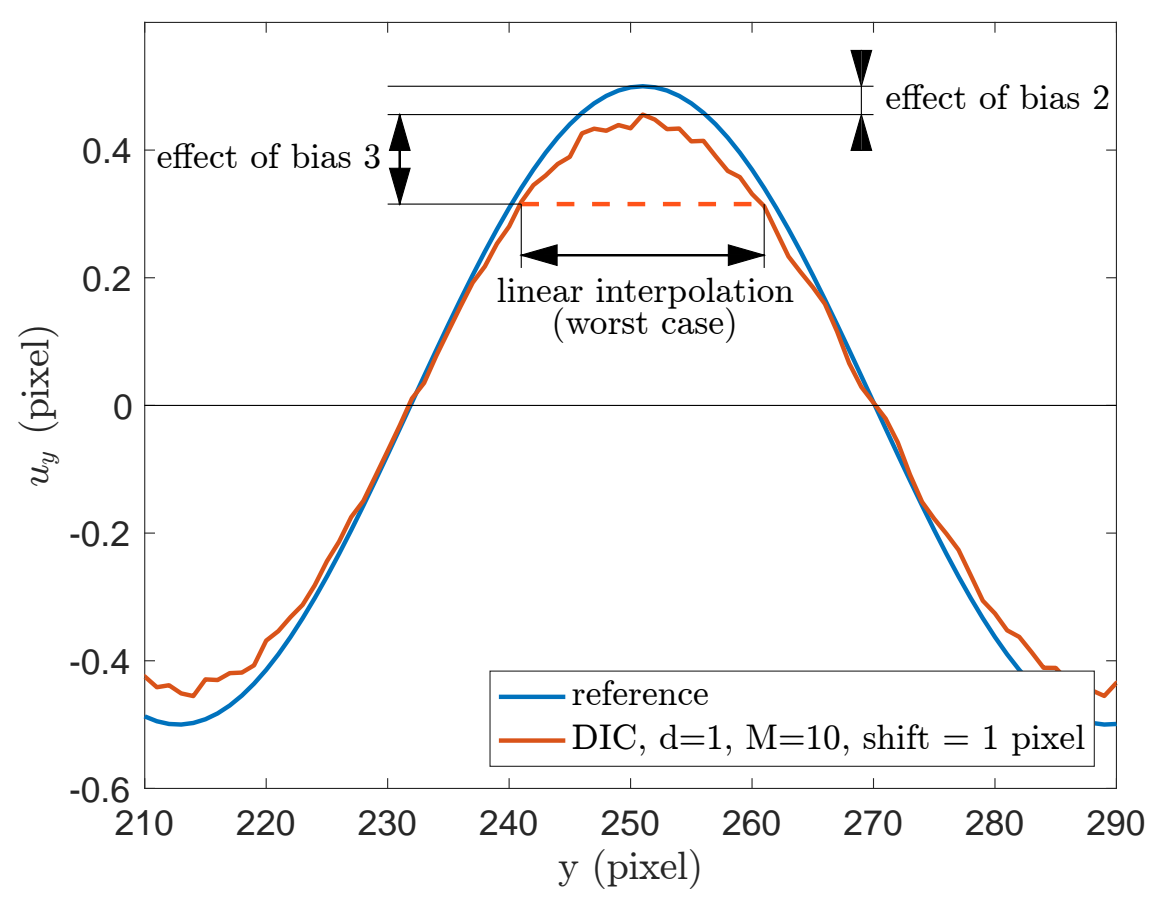

Figure 5: Effect of interpolation between remote points. Worst case of linear interpolation and maximum interpolation distance (equal to the subset size).

through this procedure since this interpolation is performed between points where the displacement is impaired by Bias 2. Bias 3 potentially affects GM if interpolation is used. In practice, however, the pixelwise displacement calculation is rapid because the calculations can easily be implemented in the Fourier domain [48], so interpolation is not necessary. It is worth mentioning that the usual WFT tools used with GM deal with dense data. Hence Bias 3 does not affect GM. To estimate the impact of Bias 3 on results obtained by DIC only, we considered the worst case, which corresponds to a linear interpolation between two adjacent subset centers at which DIC returns a displacement, i.e. the center of two adjacent subsets. The greater impact of Bias 3 is obtained when the subsets are such that they are located on both sides of an extremum of this displacement distribution, see Figure 5 . This figure represents the profile of a sinusoidal $1 \mathrm{D}$ displacement, the effect of Bias 2, which induces an attenuation of the amplitude of the displacement, and Bias 3 in the worst case. The displacement field really obtained by DIC without interpolation (thus with Bias 3 equal to zero) in superimposed. The displacement value that would be returned by DIC applied with linear interpolation would be that, in the worst case, of a point shifted by $\frac{2 M+1}{2}$ from the point where the maximum value of the theoretical displacement is reached. This displacement is equal to $A \cos \left(2 \pi f \frac{2 M+1}{2}\right)$, where $A$ is the amplitude of the sine function that is returned by DIC without interpolation, so it is also affected by Bias 2. In the same spirit as for Bias 2, if we define Bias 3 by the corresponding relative loss of amplitude of the signal, we have:

$$
\lambda_{3}=1-\cos (\pi f(2 M+1))
$$

where $2 M+1$ is the size of the subset and $f$ the frequency of the sinusoidal displacement.

Increasing Bias 3 leads to an impairment of the spatial resolution and to a decrease (thus an improvement) of the displacement resolution between the centers of the subsets, as discussed in Section 5 below. 


\subsection{Bias 4, or large-strain induced bias}

DIC is often used in cases for which large strain occurs because it is known to be an efficient tool in this case. GM is generally used in the case of small strains, so it is often thought that it is not suitable for larger ones. Indeed retrieving phases (and thus displacements) from pseudo-regular markings is made under some assumptions. In particular, it is shown in [15] that the first derivatives of the phases must be small compared to $2 \pi f$, and the second derivatives "small enough" to make Equations 17 satisfied in practice. It is clear that considering large strains may cause these assumptions not to be satisfied. Another point is that many applications of GM only deal with "small" strains, thus the phase distributions are merely subtracted and the displacement is deduced by using Equation 11 instead of Equation 12 above. This automatically induces a bias due to the fact that this subtraction is not performed at the same physical points. These points are illustrated through a simulation discussed in the following section.

The impact of large strain on DIC and GM is compared here with the following simulation. In the same spirit as for Bias 2, a vertical virtual displacement is generated, with a longitudinal strain gently evolving from 0 to $30 \%$ from the left- to the right-hand sides, with a null displacement along the centerline. This displacement field is then used to modulate the phase of a grid along $y$ using Equation 10. These reference and current grid images are then used as synthetic input data for DIC and GM. $M=10$ pixels for DIC, and a Gaussian envelope with $\sigma=5$ pixels is used here for GM.

Figure 6-a shows the reference vertical displacement field, which evolves with a linearly increasing rate when going from the left to the right. It is given here in the reference coordinate system. The displacement field retrieved by DIC is depicted in Figure 6-b. It is also given in the reference coordinate system. Since some points have moved away from the rectangular zone corresponding to the initial zone where the displacement is defined, these points are missing and the corresponding zones become therefore visible when going rightwards on the map. Applying GM with Equation 11 does not lead to the same conclusion, see Figure 6-c, since the movement of the physical points is not taken into account. This is no longer the case when considering the displacement as the solution of a fixed-point problem (Equation 12), solved iteratively by Equation 13. Figure 6-d obtained after the first iteration is similar to that shown in Figure 6-b obtained with DIC. Further iterations provide a refinement of the solution.

Bias 4 can be defined by the relative loss of amplitude of the displacement. This quantity is denoted $\lambda_{4}$. Figure 7 represents the cross-section of $\lambda_{4}$ along the line $y=400$ pixels.

The following remarks can be drawn:

- the displacement obtained by DIC perfectly matches the reference displacement since no error is detectable to the naked eye. This is an illustration that DIC is efficient in the case of large deformation;

- some slight oscillations occur on the left-hand side of the DIC curve. They are due to Bias 1 (i.e. the interpolation bias). Indeed the points along the cross section of the map at $y=400$ pixels concern subpixel displacements, which change from one column to the other because of the changing slope along the $x$-direction. The amplitude of Bias 1 is not completely negligible when $u_{y}$ is small, which explains this error on the left-hand side of the map. Bias 4 tends then to zero when $u_{y}$ increases, as expected;

- the result provided by GM by using Equation 11 is all the more biased as the displacement increases, which is logical because the movement between physical points is not taken into account. The resulting relative error is about two thirds of the slope itself with the settings used in these calculations;

- taking into account the movement of the points significantly improves the quality of the results, the relative error becoming lower than $5 \%$ for one iteration and $1 \%$ after two. No real distinction 


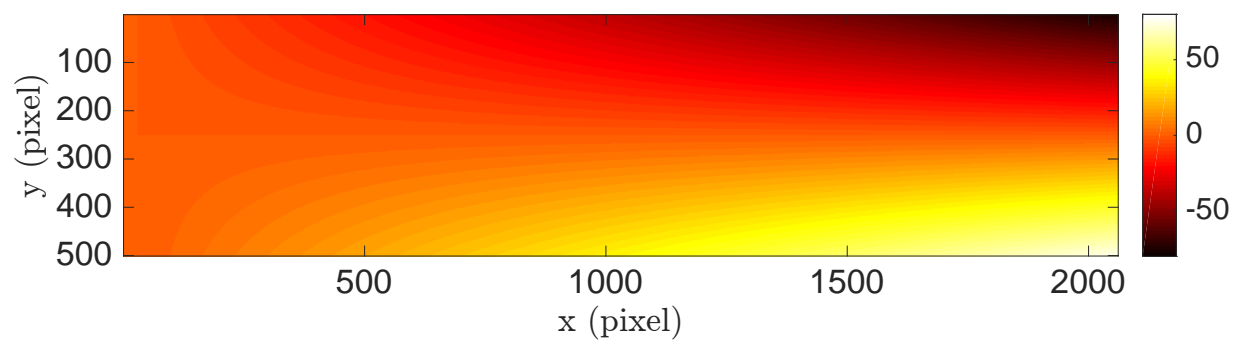

a- $u_{y}:$ reference distribution

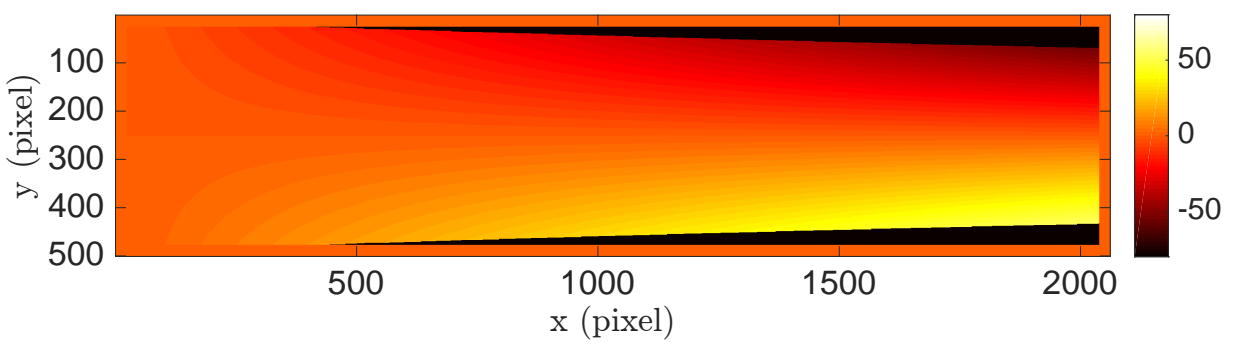

b- $u_{y}:$ map returned by DIC

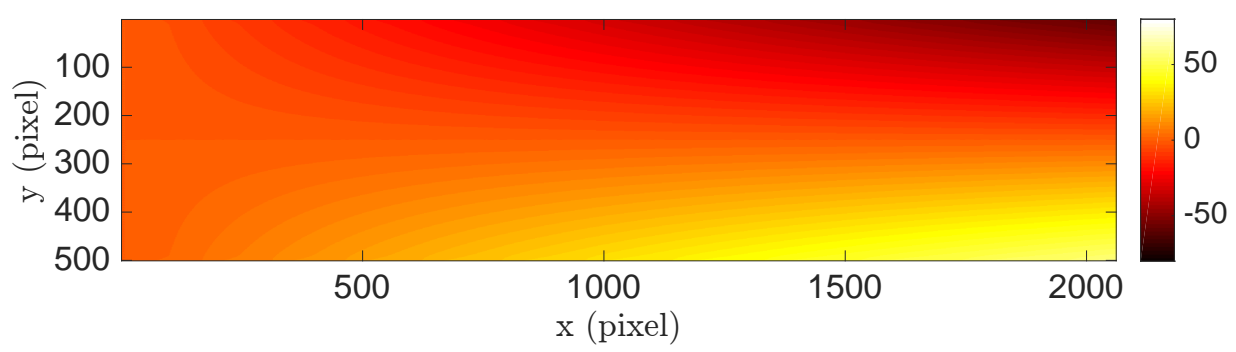

c- $u_{y}$ : map returned by GM, 0 iteration

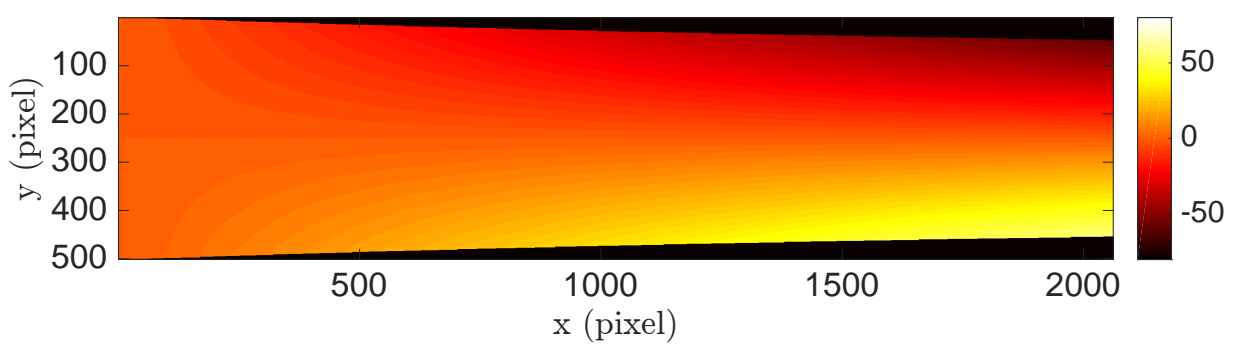

d- $u_{y}$ : map returned by GM, 1 iteration

Figure 6: a- Reference map with progressive linear displacement $u_{y}$. b- Displacement retrieved with DIC, $M=10$ pixels. c- Displacement retrieved with GM, 0 iteration. d- Displacement retrieved with GM, 1 iteration. The colorbar shows the value of the displacement $u_{y}$ (in pixel). 


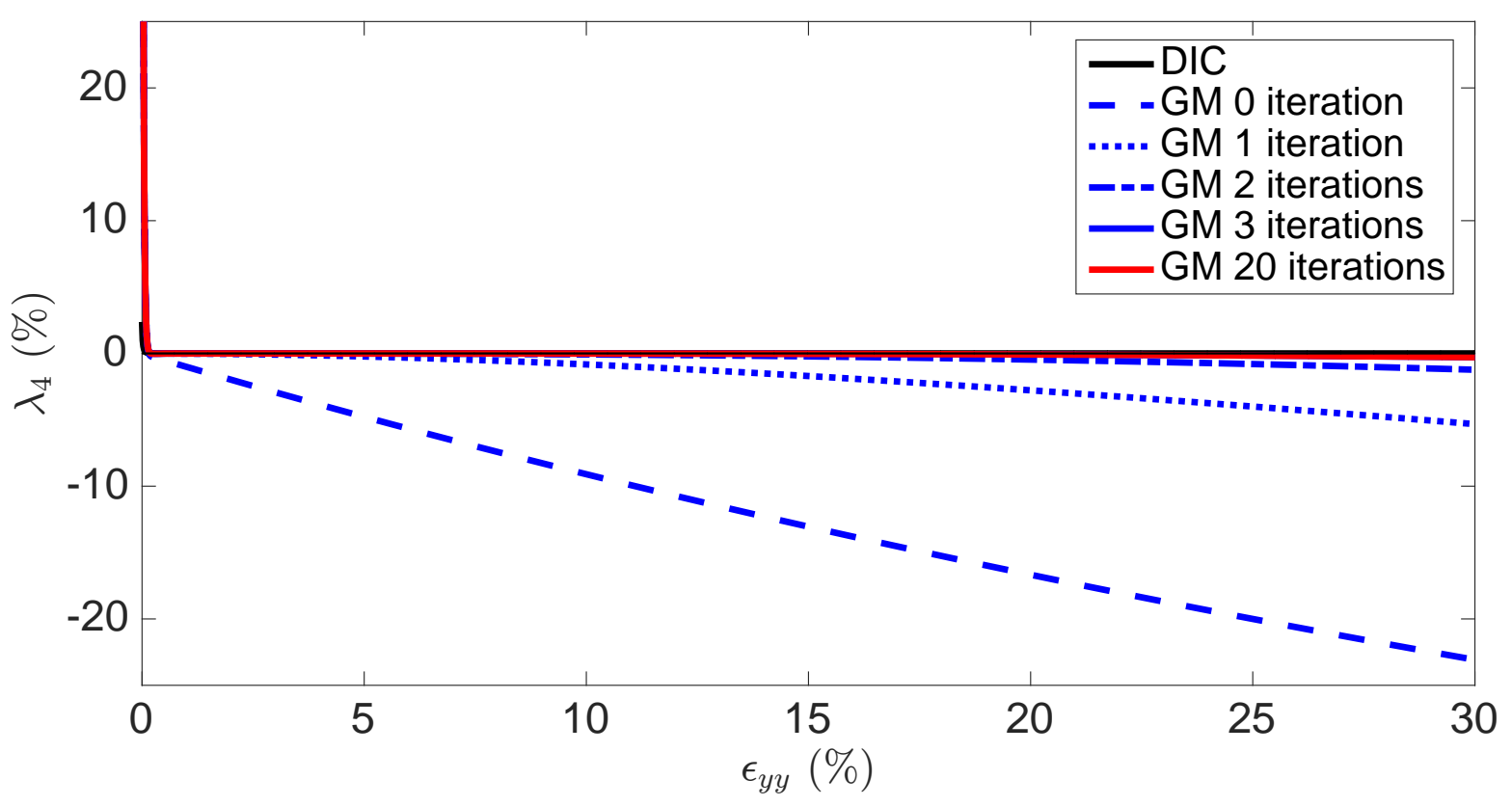

Figure 7: Bias 4 with different methods.

can be made between results obtained for three and twenty iterations, which illustrates that the iterative method based on the fixed-point algorithm quickly converges, as mentioned in [17].

In conclusion, it can be said that both DIC and GM return reliable results in the case of large strains. This remark holds at least up to the $30 \%$ considered here for GM. This is however certainly beyond the physical limit that can be reached in practice with GM, marking issues like cracking of the glue used to transfer the grid occurring earlier, as reported in [60] where nearly $18 \%$ is reached before micro-cracking damages the adhesive used to transfer the grid. Another assumption deals with the second derivative of the actual displacement, which should remain small under the analysis window for GM [15]. In practice, cracking of the painting could also potentially impair surface marking and subsequently affect displacement determination for both techniques, each in a different way. However, tackling these issues is out of the scope of this paper

\subsection{Conclusion}

The bias induced by DIC and GM was investigated in this section. The global bias is the contribution of several phenomena which have been addressed separately, and light has been shed on the difference in response of DIC and GM through various simulations. After having addressed the systematic error, the random error is investigated in the following section.

\section{Displacement resolution}

\subsection{Introduction}

The measurement resolution is defined in Ref. [27] as the smallest change in a quantity being measured that causes a perceptible change in the corresponding indication. "Perceptible" means that the quantity emerges from the noise floor, which can be quantified by the a certain multiple of the standard deviation of the noise affecting the measurement. Thus we adopt here the definition of the measurement 
resolution proposed in [61], namely the change in quantity being measured that causes a change in the corresponding indication greater than one standard deviation of the measurement noise. We consider here the noise in the displacement field which is due to camera sensor. Indeed the latter propagates to the final displacement maps through the image processing procedure which is employed. The sensor noise can be observed, ceteris paribus, by considering a stack of images of a still scene, and then observing the temporal brightness fluctuation at some pixels considered individually.

Noise in displacement maps may also have other potential causes, or may be amplified by some phenomena. Concerning DIC, overmatching causes the noise level in displacement maps returned by DIC to be amplified [59]. In the same way, the fluctuations of the marking observed with speckled patterns causes a "spatial noise" to appear. No model for this noise is available in the literature, so this noise is not considered in the present comparison based on predictive formulas experimentally validated in other studies. Quantization of the signal causes the information not to be continuous but discrete. It has been shown in [62] for GM that the effect of quantization noise on displacement/strain maps was one order of amplitude lower than that due to sensor noise. With GM only, periodicity defects in the grid deposited on the surface under investigation may induce annoying parasitic phase changes since this technique aims at detecting slight fluctuations with respect to an ideal and perfectly regular marking. Employing Equation 12 to process the phase maps with GM enables us to get rid of a significant part of the influence of these defects in the final displacement maps [54, 39]. A residue of these defects may subsist because of the interpolation needed to express the current phase distribution in the coordinate system of the reference one. This residue can be interpreted as a nonregular disturbance of the displacement field, and may potentially impact the measurement resolution. This contribution of grid defects basically depends on the printing quality of the grid, and thus cannot really be easily modeled in the general case, as for the spatial noise mentioned above for DIC.

The phenomena briefly described above are specific for each technique, so, apart from saying that they exist, they cannot be directly compared from one technique to another. Both GM and DIC are however affected by sensor noise because the latter propagates up to the final displacement maps for each technique. We propose therefore to focus only on the contribution of sensor noise to the measurement resolution for the comparison performed in this study. It means that the results presented here are partial, and that additional studies should be carried out to account for these phenomena due to marking issues, which are specific to each technique.

Concerning noise which impairs the images processed to retrieve the displacement, we assume for the sake of simplicity that it is a white noise, i.e. a homoscedastic noise with fixed standard deviation. However, noise in real camera sensors is Poisson-Gaussian and heteroscedastic (or signal-dependent), see [63] for instance. Such a noise can be changed into a homoscedastic one by using suitable numerical tools recently introduced in photomechanics, first for GM [16], then for DIC [14], but this issue is not addressed here for the sake of simplicity.

In the following, the standard deviation of the noise in images is denoted $\sigma_{\text {image }}$, and the displacement resolution $\sigma_{u}^{D I C}$ and $\sigma_{u}^{G M}$ for DIC and GM, respectively. The latter quantities can be predicted using closed-form expressions which are recalled hereafter.

\subsection{Digital Image Correlation}

Concerning DIC, $\sigma_{u}^{D I C}$ is deduced from the covariance matrix for the noise in displacement maps. Various predictive equations are available for this covariance matrix. A general formula, which takes into account the subpixel interpolation (the one which induces Bias 1), is given in $[13,14]$ :

$$
\boldsymbol{\Sigma}_{u}^{(1)}=\sigma_{\text {image }}^{2}\left(\boldsymbol{M}_{\Omega}^{-1} \boldsymbol{L}_{\Omega}\right)\left(\boldsymbol{I}+\boldsymbol{P} \boldsymbol{P}^{t}\right)\left(\boldsymbol{M}_{\Omega}^{-1} \boldsymbol{L}_{\Omega}\right)^{t}
$$

where $\boldsymbol{P}$ is the subpixel interpolation matrix. If the subpixel interpolation is not taken into account, this matrix writes as follows $[64,31,55,5]$ : 


$$
\boldsymbol{\Sigma}_{u}^{(2)}=2 \sigma_{\text {image }}^{2} \boldsymbol{M}_{\Omega}^{-1}
$$

The other matrices involved in the two preceding equations are defined by Equations 6 . The equation can be simplified further, which gives directly an upper bound for the displacement resolution $\sigma_{u}^{D I C}[31]$ and gives a better understanding of the parameters involved in the displacement resolution:

$$
\sigma_{u}^{D I C}=\sqrt{2} \frac{\sigma_{\text {image }}}{\bar{\nabla}}
$$

where $\bar{\nabla}$ is the average value of the norm of the gradient calculated over the subset. Equation 24 gives the same prediction as Equation 23 when the subpixel displacement is null, and lower values for non-zero values. We consider here Equation 23 in our predictions to have a more realistic value for the noise in the final displacement map. Since this prediction depends on the sub-pixel displacement, we consider each time the equivalent standard deviation over the specimen.

Interpolating the displacement from the centers of the subsets to pixel coordinates also impacts this results. This interpolation affects indeed the covariance matrix, which becomes in this case, when considering Equation 23 for the prediction of the covariance matrix at the center of the subsets [13]:

$$
\boldsymbol{\Sigma}_{\boldsymbol{u}}^{(3)}=\boldsymbol{C} \boldsymbol{\Sigma}_{u}^{(1)} \boldsymbol{C}^{t}
$$

where $\boldsymbol{C}$ is the matrix defining the displacement interpolation:

$$
\underline{u}_{\text {map }}=\boldsymbol{C} \underline{u}_{\text {centers }}
$$

Vector $\underline{u}_{\text {centers }}$ gathers the displacement at the centers of the subsets, while $\underline{u}_{\text {map }}$ corresponds to the pixel displacements. Again, Equation 26 gives lower values for the displacement resolution than Equation 24 for pixels located between subset centers, and the same values at the pixels defining the subset centers. This is discussed later.

\subsection{Grid Method}

Concerning GM, the covariance matrix has been completely characterized in [15] in the case of a Gaussian envelope and results obtained with other envelopes were given later in [18]. In the current cases of the Gaussian and bitriangular envelopes, we have for a grid of pitch $p$ :

$$
\left\{\begin{array}{l}
\sigma_{u}^{G M}=\frac{p}{4 \pi^{\frac{3}{2}} K \sigma} \times \sigma_{\text {image }} \text { for a Gaussian window of standard deviation } \sigma \\
\sigma_{u}^{G M}=\frac{p}{3 \pi K a} \times \sigma_{\text {image }} \text { for a triangular window of width } 2 a
\end{array}\right.
$$

where $K$ is the modulus of $\Psi(x, y, 0)$ or $\Psi\left(x, y, \frac{\pi}{2}\right)$, these two quantities being nearly equal for a regular marking like a grid. This modulus is proportional to the lighting amplitude. It is also all the higher as the line profile of the lines of the grid is "close" to a pure sine function [16].

\subsection{Results and discussion}

In the preceding results, $\sigma_{u}^{D I C}$ and $\sigma_{u}^{G M}$ are both proportional to $\sigma_{\text {image. This latter quantity is }}$ however a characteristics of the camera itself, so it is relevant to introduce the normalized displacement resolution $\widetilde{\sigma}_{u}^{D I C}$ and $\widetilde{\sigma}_{u}^{G M}$ defined by 


$$
\left\{\begin{array}{l}
\widetilde{\sigma}_{u}^{D I C}=\frac{\sigma_{u}^{D I C}}{\sigma_{\text {image }}} \\
\widetilde{\sigma}_{u}^{G M}=\frac{\sigma_{u}^{G M}}{\sigma_{\text {image }}}
\end{array}\right.
$$

$\widetilde{\sigma}_{u}^{D I C}$ and $\widetilde{\sigma}_{u}^{G M}$ can be interpreted as the coefficients of amplification of the camera sensor noise for DIC and GM, respectively. With these notations, the unit of the $\sigma_{u}^{i}, i=G M, D I C$ is pixel, and the unit of $\widetilde{\sigma}_{u}^{i}, i=G M, D I C$ is pixel/DN, where DN, which stands for Data Number, is the pseudo-unit for the gray level, as suggested in [28].

Predicting the displacement resolution for DIC needs to estimate matrix $\boldsymbol{M}_{\Omega}$ involved in Equation 23. For GM, $K$ is required. Comparing $\widetilde{\sigma}_{u}^{D I C}$ and $\widetilde{\sigma}_{u}^{G M}$ from properties of $\boldsymbol{M}_{\Omega}$ and $K$ seems not obvious, so a simple procedure consists of performing this comparison again with the same set of two images processed by both techniques separately. A grid image is therefore considered once again (random patterns are discussed at the end of the paper). The very nature of the displacement between the two images has no impact on the estimates which are used to assess the displacement resolution, so the same set of images already considered in Section 3.4 were processed here for the sake of convenience. The calculations were performed for five subset sizes $2 M+1(M=3,6,10,15,20$ pixels $)$ and with a bilinear matching function $(d=1)$ for DIC. Three sizes of the Gaussian and triangular kernel were employed for GM ( $\sigma=a=5,10,15$ pixels). Figure 8 shows the average of the normalized displacement resolution estimated for these different settings. Concerning the order of magnitude of the standard deviation for the noise, it is worth remembering that the simulations presented here were carried by using grid images, which were obtained with a gray depth equal to 12 bits. Consequently, $\tilde{\sigma}_{u}$ shall be multiplied by a value for $\sigma_{\text {image }}$ expressed with the same gray depth to be consistent. For readers having in mind values for $\sigma_{\text {image }}$ obtained with their own camera featuring a gray depth equal $n=8,12,14$ or $n=16$ bits, which are usual values for DSLR (Digital Single Lens Reflex) or professional cameras, $\sigma_{u}$ is obtained by multiplying $\widetilde{\sigma}_{u}$ in Figure 8 successively by $\sigma_{i m a g e}$ and by $2^{(12-n)}$ to deduce a correct estimate of $\sigma_{u}$. For instance, for a 8-bit camera characterized by $\sigma_{\text {image }}=2 \mathrm{DN}$, the corresponding value for $\sigma_{u}$ is $\sigma_{u}=\widetilde{\sigma}_{u} \times 2 \times 2^{4}=\widetilde{\sigma}_{u} \times 2^{5}$ (pixels). The same remark holds for the following figures where $\widetilde{\sigma}_{u}$ is involved (Figures 9-12)).

It can be seen in Figure 8 that the displacement resolution decreases as the size of the subset or the window increases, which is logical since the averaging effect on the noise is more pronounced as the number of pixels considered to calculate the displacement at any pixel increases. The second remark is that the order of magnitude of the different values obtained for the displacement resolution is the same for both techniques. It is, however, difficult to go further with the comparison between the techniques since the obtained results depend on settings chosen by the user for each technique separately, more precisely on the size of the subset for DIC and window for GM. Those sizes only constitute a rough parameter to compare the techniques. In addition, the Gaussian curve has no compact support, so the size is only arbitrarily defined using the $3-\sigma$ rule. Performing a fair comparison thus requires to introduce another parameter, namely the spatial resolution, which must be defined on the same basis for both techniques. This point is investigated in the following section.

\section{$5 \quad$ Link between Bias 2, Bias 3 and measurement resolution through the spatial resolution}

The objective here is to examine how to compare the performance of DIC and GM with the same definition of the spatial resolution for both techniques. We first show how to determine this spatial resolution for DIC and GM. Then we examine how the displacement resolution depends on the spatial 


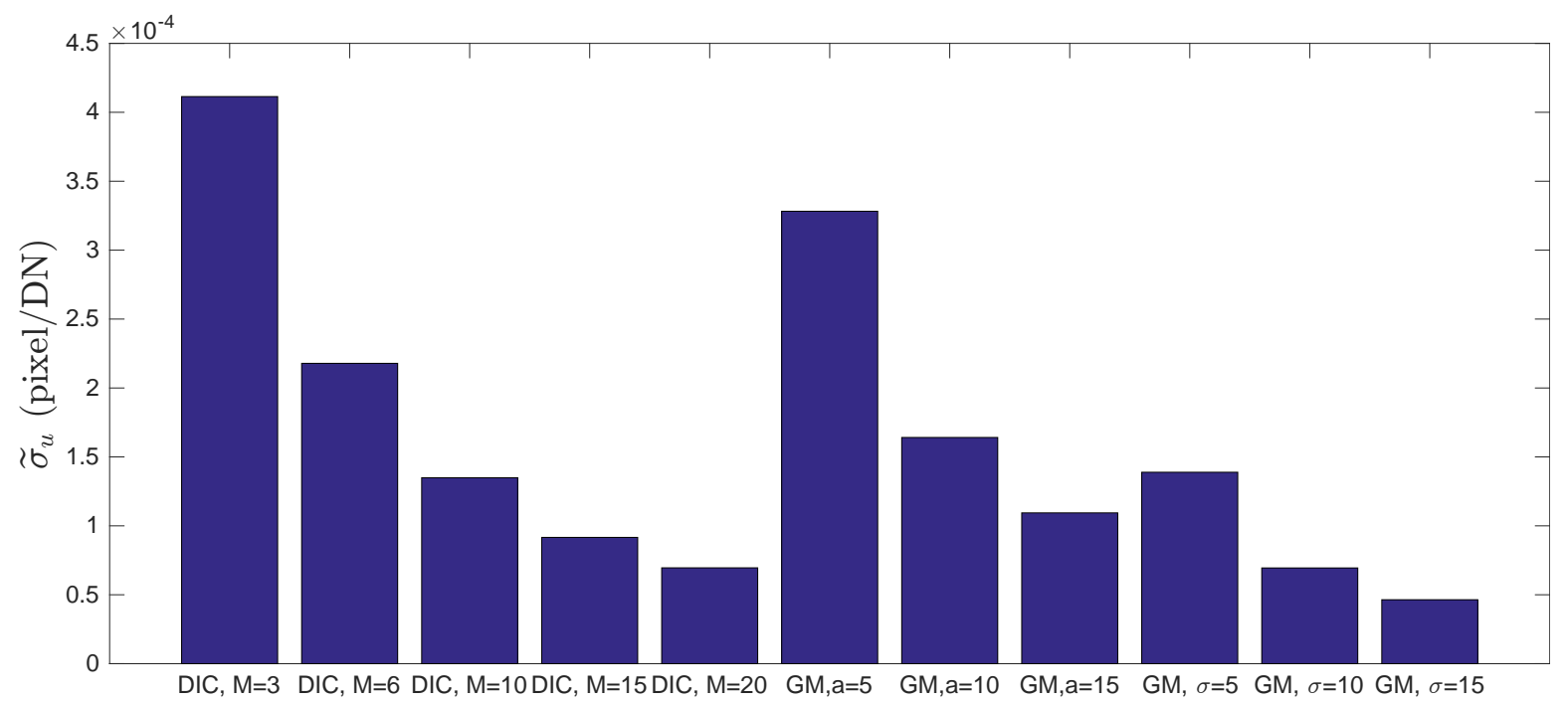

Figure 8: Normalized displacement resolution (upper bound for DIC). The values reported in this figure are obtained with a gray depth equal to $12 \mathrm{bits}$, so $\sigma_{\text {image }}$ shall be estimated accordingly to obtain a consistent value of $\sigma_{u}$ by multiplying $\widetilde{\sigma}_{u}$ by $\sigma_{\text {image }}$.

resolution for both techniques, and consider the product between these two quantities to compare DIC and GM. Finally, we discuss the influence of Bias 3 on the results when interpolation between remote points is performed, which is generally the case with DIC.

\subsection{Determination of the spatial resolution for DIC}

As mentioned in Section 2.3, the spatial resolution reflects the ability of a technique to distinguish close features in a map. This quantity depends on Bias 2 and even on Bias 3 when interpolation between remote points is employed. We propose here to define the spatial resolution as the period of the sine displacement beyond which the relative loss in amplitude of the displacement returned by the measuring technique is greater than a certain value ([11] after similar definition proposed in [65]). If no interpolation is used and regardless of noise, this relative loss is directly $\lambda_{2}$ defined above. If interpolation is used, this is $1-\left(1-\lambda_{2}\right)\left(1-\lambda_{3}\right)$.

Let us first consider the case $\lambda_{3}=0$ (no interpolation between remote points). As in previous studies [11], it is proposed first to fix Bias 2 to a certain acceptable value, here $20 \%$. The spatial resolution is then deduced from the transfer functions discussed in Section 3.2 for each setting retained for the calculation (subset and window sizes). The equation $1-\widehat{h}_{M}(\widetilde{k})=\lambda_{2}=0.2$ is solved numerically with Matlabß for the SG filter, no analytical solution being available. It means that the normalized wave length $\widetilde{k}=\widetilde{k}_{0}$ solution of the following equation (see Equation 20 where $\widehat{h}_{M}(\widetilde{k})$ is defined)

$$
1-h(0)-2 \sum_{i=1}^{M} h(i) \cos (i \widetilde{k} \pi)=\lambda_{2}=0.2
$$

must first be determined. The spatial resolution $d_{u}$ is then deduced. This is merely

$$
d_{u}=\frac{2}{\widetilde{k}_{0}}
$$

since the Nyquist frequency is equal to 0.5 pixel $^{-1}$. As explained in Section 3.2.3, the $h(i)$ coefficients, $i\{0 \cdots M\}$, represent the value of the filter used for modeling Bias 2 for DIC, namely the SG filer. 
Again, this filter is sampled over $2 M+1$ points, where $M=3,6,10,15,20$ pixels. This calculation enables us to determine $d_{u}$ for DIC. The associated displacement resolution is then deduced using the same approach as that shown in Section 4.

\subsection{Determination of the spatial resolution for GM}

Concerning GM, two methods are available to determine the spatial resolution since one can consider either the continuous or the discrete version of the transfer function, for both the Gaussian and the triangular windows.

\subsubsection{With the closed-form expression of the bias}

For the continuous approach and after [17] and [18], the relationship between spatial resolution $d_{u}$ and $\lambda_{2}$ writes as follows:

$$
\begin{aligned}
& \lambda_{2}=1-e^{-2 \pi^{2} \frac{\sigma^{2}}{d_{u}^{2}}} \text { for a Gaussian window of standard deviation } \sigma \\
& \lambda_{2}=1-\operatorname{sinc}^{2}\left(\frac{a}{d_{u}}\right) \text { for a triangular window of width } 2 a
\end{aligned}
$$

In practice, the bias can be considered as a parameter fixed by the user (here 20\%). The spatial resolution can thus be deduced from Equations 32 in which $d_{u}$ is the unknown, all other quantities involved in this equation being known. In the case of a Gaussian window, Equation 32 leads to

$$
d_{u}=\frac{\sqrt{2} \pi \sigma}{\sqrt{-\log \left(1-\lambda_{2}\right)}} \quad \text { with } 0<\lambda_{2}<1
$$

\subsubsection{Discrete transfer function of the Gaussian filter}

In Section 3.2, we introduced the discrete version of the transfer function for both the Gaussian and the triangular filters to make possible the comparison between DIC and GM. We can therefore consider the same approach here, by taking Equation 30 in which the $h(i)$ coefficients, $i \in\{0, \cdots, M\}$, represent now the value of either the Gaussian or the triangular filter for the different values of $i$. The benefit is to have the same approach for DIC and GM to determine $d_{u}$, namely the discrete one. However it can be checked that both the continuous and the discrete approaches lead to practically the same results for the Gaussian envelop, so the continuous approach is employed in this case hereafter for the sake of convenience, a closed-form solution being available for the Gaussian envelope.

\subsection{Normalized measurement resolution as a function of spatial resolution for DIC and GM}

For DIC, the spatial resolution is determined for a given subset size using the approach given in Section 5.1. The displacement resolution is obtained for the same subset size using the procedure given in Section 4.2. This enables us to have a representative point for DIC for each subset size in the spatial resolution-displacement resolution plane.

For GM, we can follow the same procedure as for DIC, i.e. estimating the spatial resolution with the procedure given in Section 5.1 (continuous case) and then estimating the displacement resolution given in Section 4.3, which gives a representative point for GM for each type of window and for each size in the same plane as above. In the particular case of a Gaussian envelope, we can also apply the following predictive formula given in [17], deduced from Equations 29 and 34: 


$$
\widetilde{\sigma}_{u}^{G M}=\frac{p}{2 \sqrt{2 \pi} d_{u} K \sqrt{-\log \left(1-\lambda_{2}\right)}} \quad \text { with } 0<\lambda_{2}<1
$$

where

- $p$ is the pitch of the grid;

- $K$ is the modulus of the WFT of the grid image. The modulus obtained for the WFT calculated along $x$ or $y$ are theoretically equal for a regular grid [15], so no distinction is made here in the notation for values of $K$ calculated along $x$ and $y$.

Choosing a value for $\lambda_{2}$ gives in turn a spatial resolution $d_{u}$ which depends on the size of the window chosen for the calculations. The displacement resolution $\widetilde{\sigma}_{u}$ is eventually expressed as a function of $d_{u}$. The corresponding points are shown in Figure 9. The conclusion is similar to that drawn from the preceding results: as expected, the displacement resolution decreases for both techniques as the spatial resolution increases, but DIC and GM are now fairly comparable. We can see that triangular and Gaussian windows seem to be nearly on the same curve, while the points reflecting the response of DIC are slightly above those of GM.

This figure illustrates the tradeoff between displacement resolution and spatial resolution that every user of full-field measurement method faces in one way or another. The ability of each technique to manage at best this tradeoff, namely having at the same time the lowest spatial resolution and the lowest measurement resolution as possible, is reflected by the following product

$$
\tilde{\alpha}=d_{u} \times \widetilde{\sigma}_{u},
$$

which corresponds to the area of the rectangles constructed between the origine of the plane and any point in the diagram (see Figure 9). We propose to name this product the normalized metrological efficiency indicator for a given value of $\lambda_{2}$. It is worth remembering that in this study, this product does not take into account the "spatial noise" due to marking issues which are specific to each technique. In the same spirit, the product defined by

$$
\alpha=d_{u} \times \sigma_{u}
$$

is the metrological efficiency indicator. This quantity should be as low as possible to reflect a good compromise between spatial resolution and displacement resolution for a given bias. As explained in [17] in the case of a Gaussian envelope, $\tilde{\alpha}$ is constant for GM for a given bias whatever the size of the analysis window used in the WFT. This can also easily be deduced from Equation 35. This property is less clearly established in the case of the triangular envelope, $d_{u}$ being obtained from numerical calculations. $\tilde{\alpha}$ is also constant for DIC. We can therefore calculate $\tilde{\alpha}$ in all these cases and compare the results which are obtained. This area of the aforementioned rectangles is reported in Figure 10.

We can see in this figure that $\widetilde{\alpha}$ is constant in all cases, thus it really constitutes an indicator to compare techniques among themselves. Concerning GM, $\widetilde{\alpha}$ is nearly the same for the two types of windows considered here. Another conclusion is that this product is slightly greater for DIC than for GM, which means that at least for the present image, the compromise between displacement resolution and spatial resolution is not as good as that obtained for GM, but the difference between the two is not very marked.

\subsection{Impairment of the performance of DIC due to Bias 3}

The first results given above for DIC rely on the particular case of a 1-pixel subset shift. Such a small shift is generally not adopted in practice because of the high computational cost which is induced, 


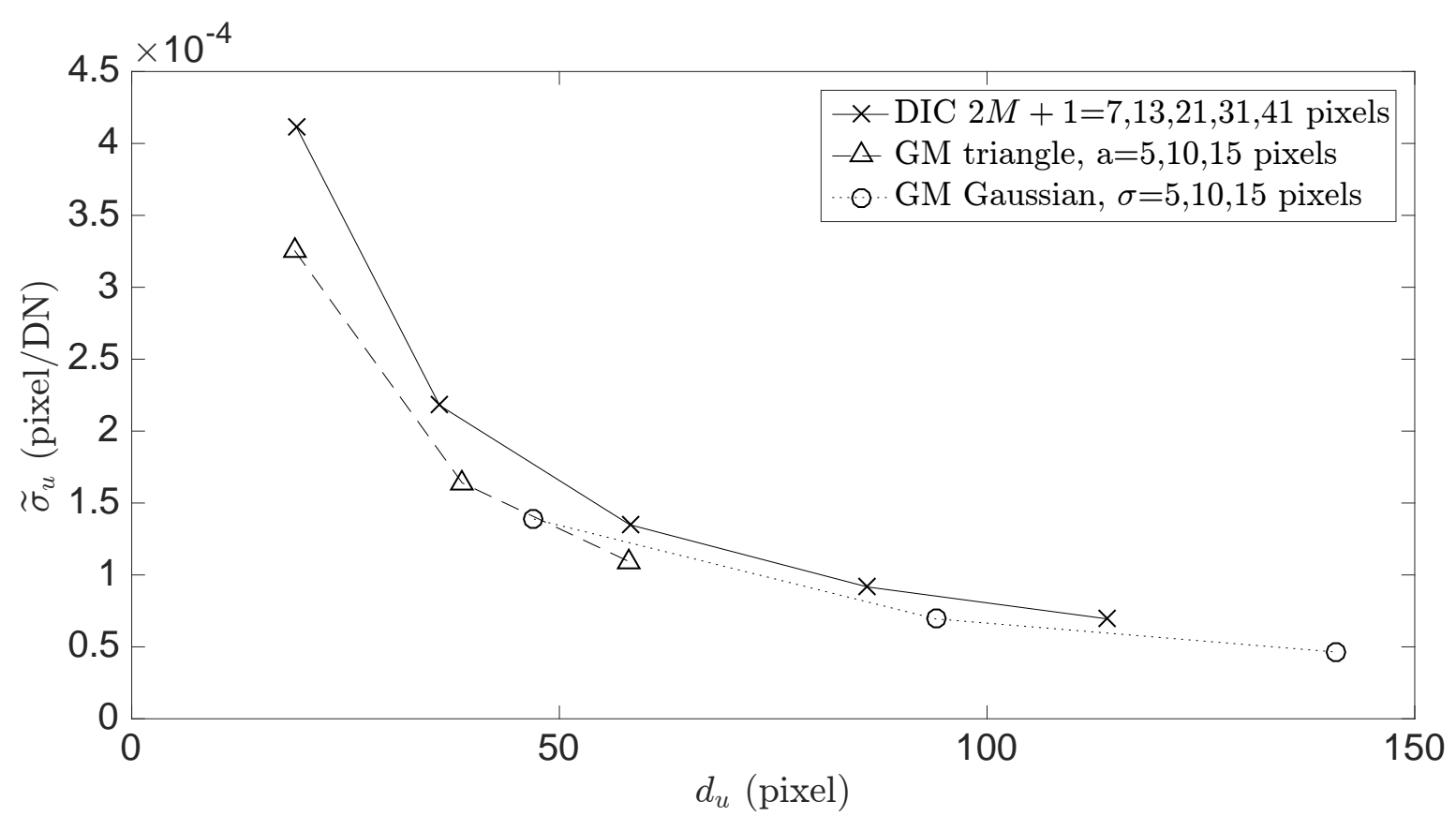

a- $\widetilde{\sigma}_{u}$ as a function of $d_{u}$

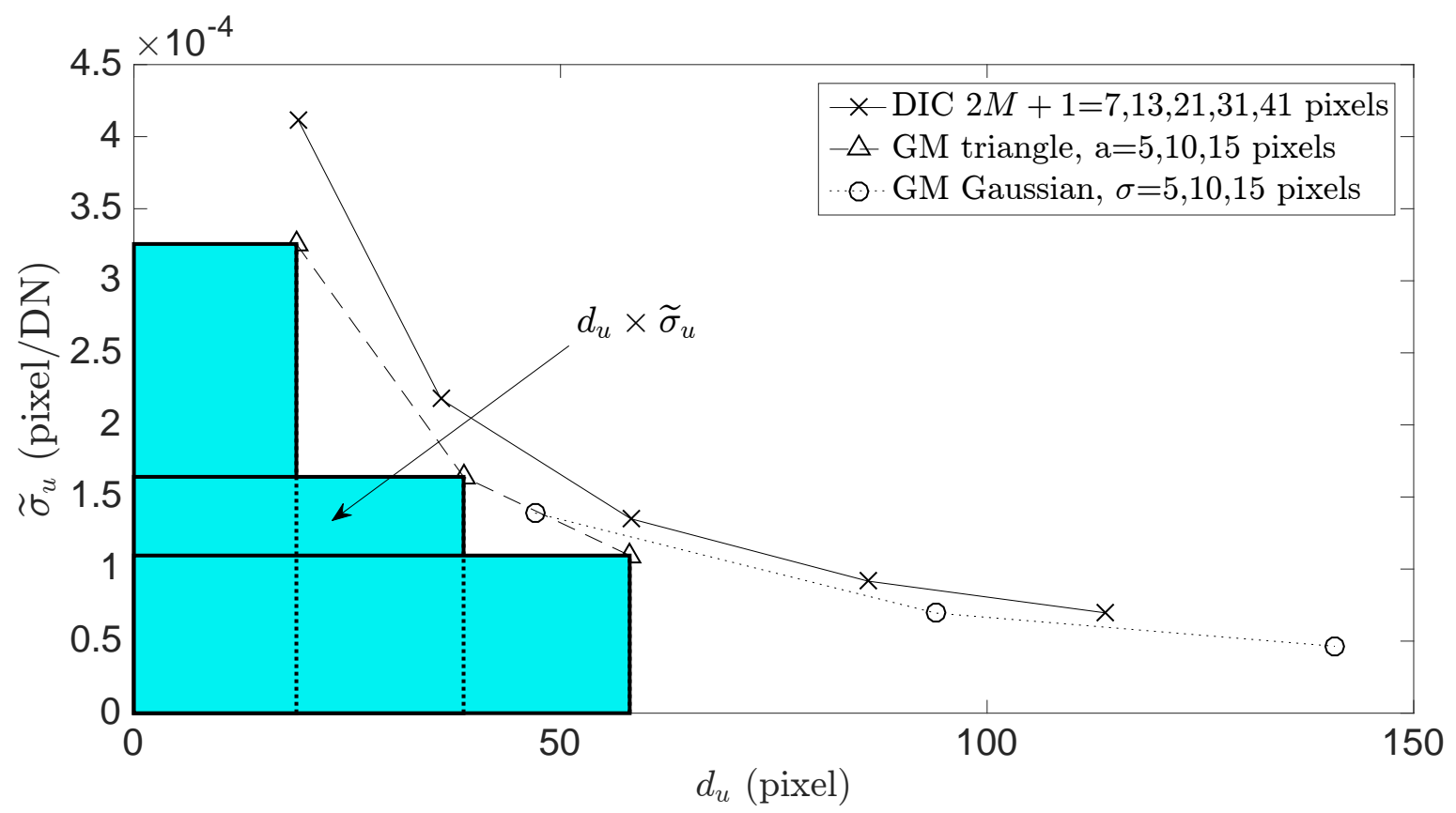

b- construction of the rectangles

Figure 9: Normalized displacement resolution as a function of the displacement resolution. DIC: $M=3,6,10,15,20$ pixels, GM triangle: $a=5,10,15$ pixels, GM Gaussian: $\sigma=5,10,15$ pixels. The values reported in this figure are obtained with a gray depth equal to 12 bits, so $\sigma_{\text {image }}$ shall be estimated accordingly to obtain a consistent value of $\sigma_{u}$ by multiplying $\widetilde{\sigma}_{u}$ by $\sigma_{\text {image }}$, as explained in Section 4.4. 


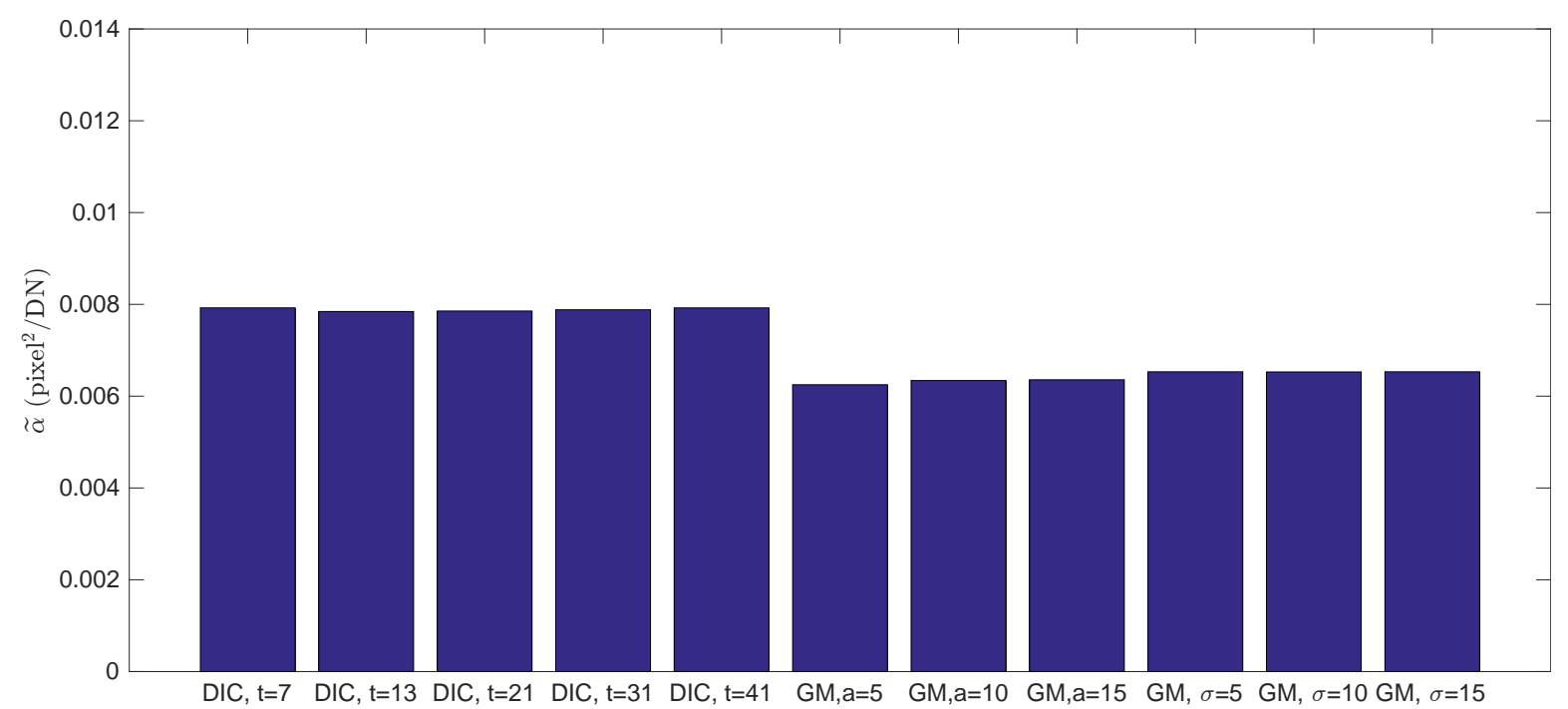

Figure 10: DIC and GM: $\tilde{\alpha}$ for various settings. The values reported in this figure are obtained with a gray depth equal to 12 bits, so $\sigma_{\text {image }}$ shall be estimated accordingly to obtain a consistent value of $\sigma_{u}$ by multiplying $\widetilde{\sigma}_{u}$ by $\sigma_{\text {image }}$.

as discussed in the section dealing with Bias 3 above. Since almost all DIC codes introduce higher subset shift, the output in terms of full-field displacement maps are interpolated values. It is therefore important to highlight the effect of Bias 3 for DIC.

Effect on the spatial resolution The impact of the additional bias due to interpolation can be estimated using the same approach as in the preceding section. Figure 5 shows indeed that the worst situation corresponds to the case for which two remote points located on both sides of the maximum of a sine displacement distribution are linearly interpolated. The relative loss of amplitude for the displacement returned by DIC after interpolation is in this (worst) case: $1-\left(1-\lambda_{2}\right)\left(1-\lambda_{3}\right)$. This leads to a new value for the spatial resolution, which is greater than that obtained when Bias 2 is considered alone. In the same spirit as for $d_{u}$ calculated in the case "without interpolation", the normalized wave number $\tilde{k}_{0}$ for which $1-\left(1-\lambda_{2}\right)\left(1-\lambda_{3}\right)$ becomes equal to 0.2 must first be determined. Thus the solution of Equation 38 below

$$
1-\left(1-\cos \left(\pi \frac{\widetilde{k}}{2}(2 M+1)\right)\right)\left(1-h(0)-2 \sum_{i=1}^{M} h(i) \cos (i \widetilde{k} \pi)\right)=1-\left(1-\lambda_{2}\right)\left(1-\lambda_{3}\right)=0.2
$$

must now be considered instead of the solution of Equation 30. This solution is found numerically, the calculations being again not tractable. Then the corresponding spatial resolution, denoted now $d_{u}^{\prime}$, is deduced again from Equation 31 .

Effect on the displacement resolution The displacement resolution in this case has a new feature compared to the displacement resolution obtained without Bias 3: since the displacement at the center of the subsets are interpolated, the displacement resolution decreases in between (which means that it is improved). This is a consequence of Equation 26 above (see $[13,14]$ for more details). This lower value is denoted $\widetilde{\sigma}_{u}^{\prime}$. At the centers of the subsets, however, the displacement resolution remains unchanged compared to the values discussed in the preceding case because interpolation has no effect at these points. Consequently, the spatial resolution is the highest when the displacement resolution is the 


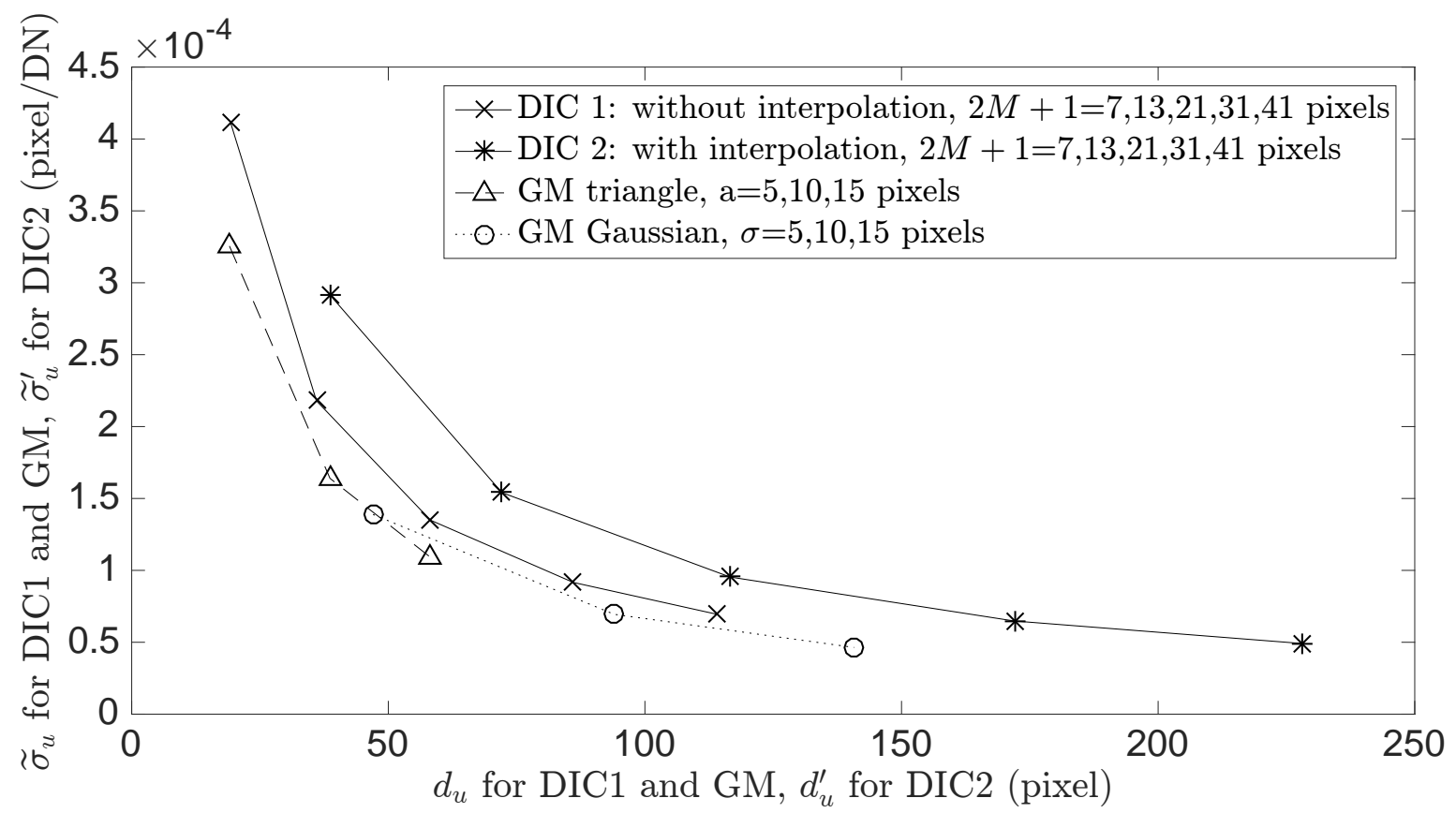

Figure 11: Effect of Bias 3 due to interpolation between remote points. Normalized displacement resolution $\widetilde{\sigma}_{u}$ as a function of the spatial resolution $d_{u}$ (GM) or $d_{u}^{\prime}$ (DIC). For DIC, Bias 3 impairs the spatial resolution but improves the measurement resolution. As a result $\widetilde{\alpha}$ is greater, so the compromise between measurement resolution and spatial resolution is impaired. The values reported in this figure are obtained with a gray depth equal to $12 \mathrm{bits}$, so $\sigma_{\text {image }}$ shall be estimated accordingly to obtain a consistent value of $\sigma_{u}$ by multiplying $\widetilde{\sigma}_{u}$ by $\sigma_{\text {image }}$.

lowest and vice versa, which means that both parameters fluctuates throughout a given displacement map.

Figure 11 summarizes these results. The points representing the response of DIC move to the right when interpolation is taken into account, showing that the spatial resolution is impaired. At the same time, these points move however downwards, illustrating that the displacement resolution is improved. The points corresponding to GM do not change because $\lambda_{3}=0$ for GM, calculations being performed pixelwise.

Effect on the compromise between displacement resolution and spatial resolution The compromise between displacement resolution and spatial resolution can again be represented by the product between these two quantities, denoted here

$$
\widetilde{\alpha}^{\prime}=d_{u}^{\prime} \times \widetilde{\sigma}_{u}^{\prime}
$$

The new values for DIC are represented in Figure 12 along with the points of GM. It can be seen that interpolation negatively impacts this compromise when comparing these values to those shown in Figure 10. Interestingly $\widetilde{\alpha}^{\prime}$ remains constant for DIC in this case too. The main remark is that Bias 3 severely affects the compromise between displacement resolution and spatial resolution, $\widetilde{\alpha}^{\prime}$ being nearly $50 \%$ greater than $\widetilde{\alpha}$ calculated without Bias 3 


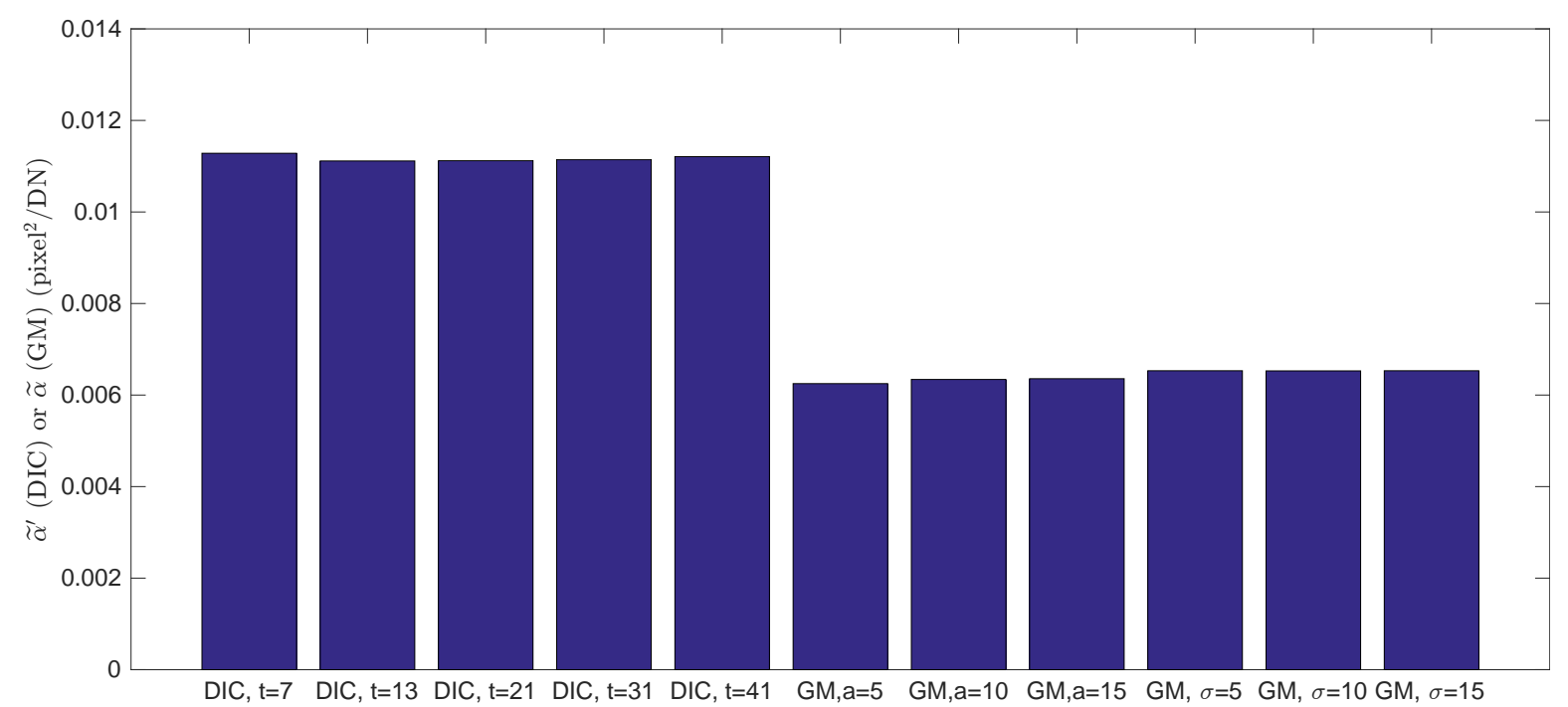

Figure 12: $\widetilde{\alpha}^{\prime}$ (DIC) and $\widetilde{\alpha}(\mathrm{GM})$ for various settings. The values reported in this figure are obtained with a gray depth equal to $12 \mathrm{bits}$, so $\sigma_{\text {image }}$ shall be estimated accordingly to obtain a consistent value of $\sigma_{u}$ by multiplying $\widetilde{\sigma}_{u}$ by $\sigma_{\text {image }}$.

\section{From regular to random patterns}

\subsection{Introduction}

The reader accustomed to using DIC is certainly wondering how to establish a link between the results presented above obtained with grid images processed by DIC, and results that would be obtained with "usual" speckled patterns. Indeed DIC also works with regular patterns like grids, which enabled us to stabilize the influence of the pattern itself in the analysis presented in the previous sections. However, such regular patterns are scarcely employed with DIC, mainly because depositing such patterns is less convenient than depositing a random pattern. We propose here to compare the results obtained with random patterns and grid images processed by DIC, and consequenty results obtained with random patterns processed by DIC and grid images processed by GM. Considering all the quantities introduced in the preceding sections to perform this comparison is out of the scope of this paper, so we focus mainly on the compromise between spatial resolution and displacement resolution, which has the advantage of being quantified by a product nearly independent of the size of the subset for DIC, and window for GM. Several types of markings are used for this purpose, namely a regular one and five random ones. Again, it is worth remembering that the "spatial noise" due to marking fluctuations is not taken into account in the present study. Such fluctuations are expected to be more significant for speckles than for regular grids, which justifies further work on this specific point. We compare first the results obtained by DIC and GM on the grid image. Random patterns are then considered for DIC.

\subsection{Metrological performance of DIC and GM with the same regular pattern}

We consider first the picture of a real grid shown in Figure 13-a. It is obtained with a 12-bit CCD camera but images are stored in tif files featuring a gray depth equal to 16 bits by multiplying the raw 12-bit output by $2^{4}$. Five pixels are used to discretize one grid pitch. In the same spirit as in the previous sections, the idea is to consider parameter $\widetilde{\alpha}$ for this comparison since it reflects the tradeoff between measurement and spatial resolutions. The spatial resolution $d_{u}$ only depends on the size of the 


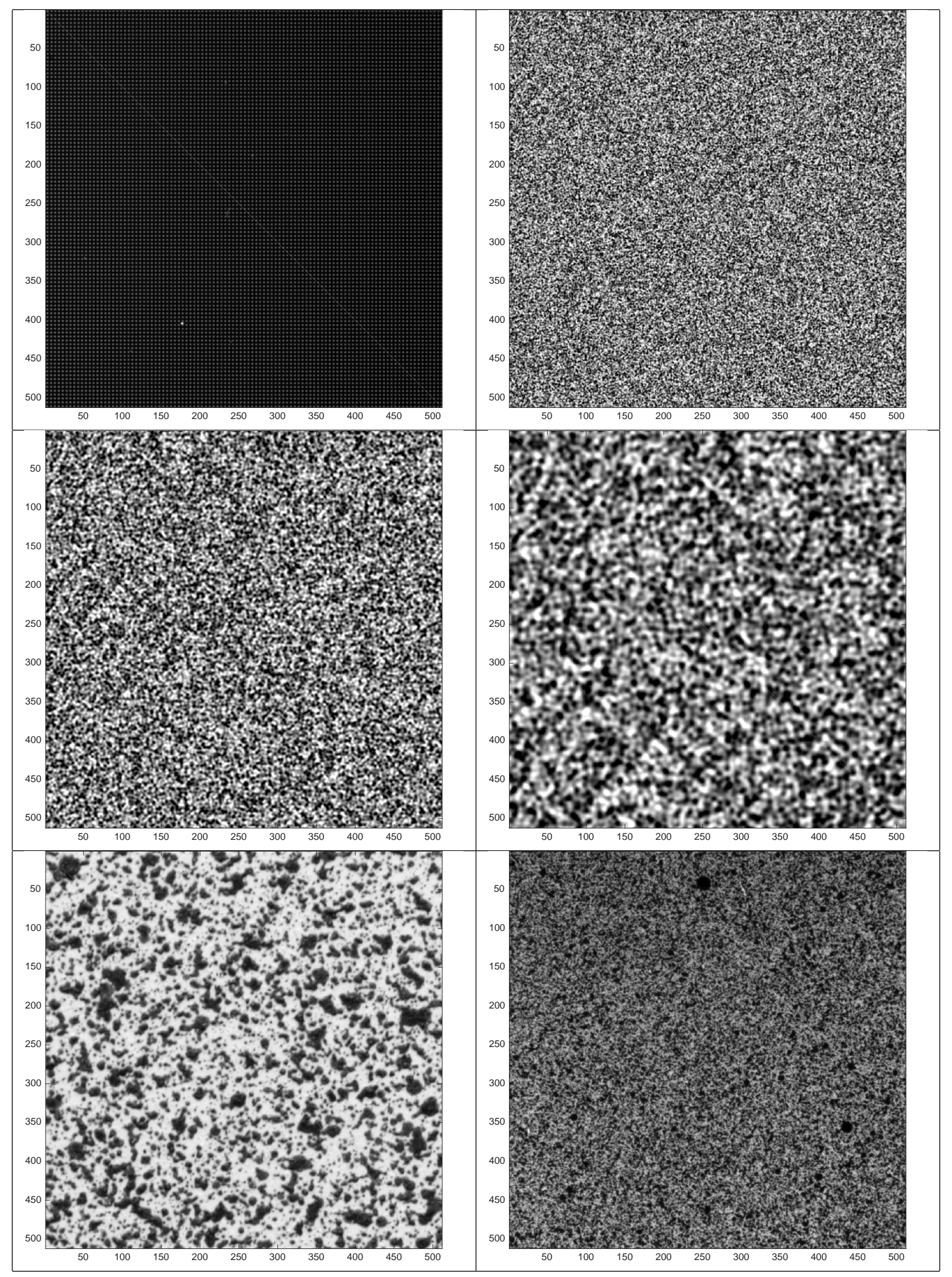

Figure 13: Grid and speckle images under study. 
subset for DIC, and on the size of the window in GM. While the normalized displacement resolution $\widetilde{\sigma}_{u}$ is defined by Equation 29, the displacement resolution $\sigma_{u}$ is estimated with Equation 26 for DIC, and with Equation 28 for GM. The obtained results are compared by calculating pixelwise the ratio $\tilde{\sigma}_{u}^{G M} / \widetilde{\sigma}_{u}^{D I C}$. DIC is performed for three values of the subset size $(2 M+1=7,13,21$ pixels $)$ and GM for three values of $\sigma(5,10,15$ pixels), a Gaussian window being used here. This makes $3 \times 3=9$ different combinations, and thus 9 different maps for this ratio. The results obtained with a triangular window are not discussed here because they lead to conclusions similar to those obtained with a Gaussian window. As explained above, the product between displacement and spatial resolution $\widetilde{\alpha}^{\prime}$ is expected to be constant for a given technique and lighting conditions. By definition, the same remark holds for the ratio defined above.

Figures 14-a shows the portion of the grid figure for which the results are considered in the comparison. This is the same as Figure 13-a, but it has been cropped to get rid of the border effects. As an illustration, the different subset sizes used here with DIC and window sizes used with GM are superimposed to Figure 14-a. It is worth remembering that the "size of the window for GM" is considered here for a Gaussian window. This is a circle whose diameter is $3 \sigma$ according to the $3-\sigma$ rule, but this size is arbitrary. The value of the spatial resolution estimated for a given value for Bias 2 equal to $20 \%$ is plotted in front of each subset/window. These spatial resolutions are estimated using the procedure given in Sections 5.1 and 5.2 above for DIC and GM, respectively.

Figures 14-b and -c show two typical examples of the spatial distribution of the ratio $\widetilde{\sigma}_{u}^{G M} / \widetilde{\sigma}_{u}^{D I C}$. They are obtained with $\sigma=5$ pixels and $M=3$ pixels and $\sigma=5$ pixels and $M=10$ pixels, respectively. It can be seen that the global aspect is the same, but the blur in this distribution is higher in the second case, which is due to the fact that local averaging is more pronounced. Interestingly, the ratio is mainly lower than one (dominant blue in the maps), which means the compromise between displacement and spatial resolutions is better for GM than for DIC. On average, the relative difference is equal to $10 \%$ in both cases. The ratio is however much greater than one on a very localized zone which corresponds to the yellow spot on the map. Comparing both maps with the grid image recalled in Figure 14-a enables us to see that this yellow spot corresponds to a localized lack of marking. Interestingly the zone affected by the defect is much smaller on the grid image than on the maps, which is a consequence of the fact that the information displayed at a given pixel of a map depends on the information contained in its surrounding pixels. Consequently, any marking defect spreads out in the maps for GM. The mean distribution is finally calculated by averaging the nine combinations between $\sigma=5,10,15$ pixels and $M=3,6,10$ pixels, which gives a clearer view on the spatial distribution of the ratio. This ratio is nearly uniformly spatially distributed apart from the yellow zone. On average, the mean ratio is equal to 0.90 , which means that the tradeoff between spatial resolution and measurement resolution is more favorable for GM than for DIC for this grid image. It is worth remembering that applying interpolation between remote points in DIC would impair the performance of DIC, and this ratio would therefore be lower.

\subsection{From regular to random patterns, impact on the metrological performance of DIC}

\subsubsection{Introduction}

The influence of the marking on the results obtained by DIC is now studied by considering the five speckle images shown in Figures 13-b to 13-f. The first three speckles are synthetic images used in [2] to compare various DIC programmes. Their gray depth is 8 bits. They are named Speckle 1 to Speckle 3 in the following. The last two are real in-house speckles obtained by painting a surface in white, and then spraying droplets of black paint. Their gray depth is 16 bits. These two speckles are named Speckle 4 and Speckle 5. Such markings can be processed only by DIC. Nevertheless the obtained results can be compared with those obtained by DIC with the grid image, and consequently 


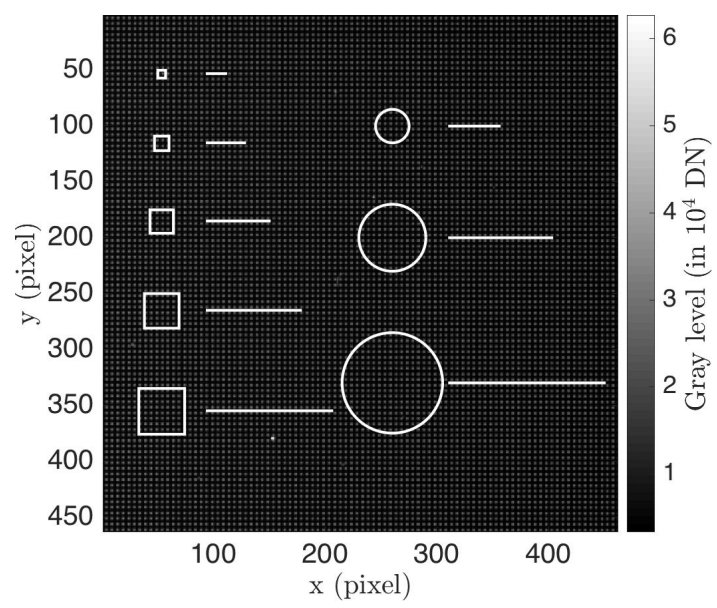

a- Grid pattern with subsets and windows used to process the image.

Corresponding spatial resolutions

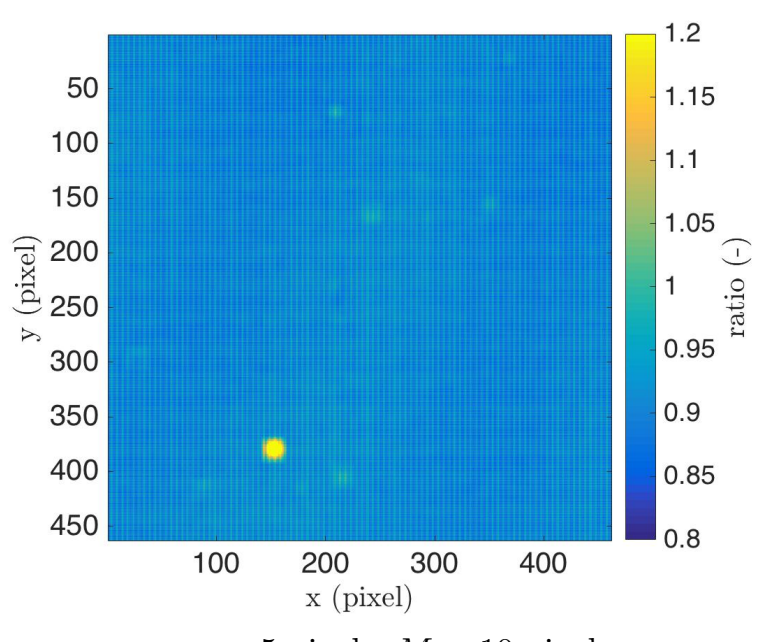

c- $\sigma=5$ pixels, $M=10$ pixels

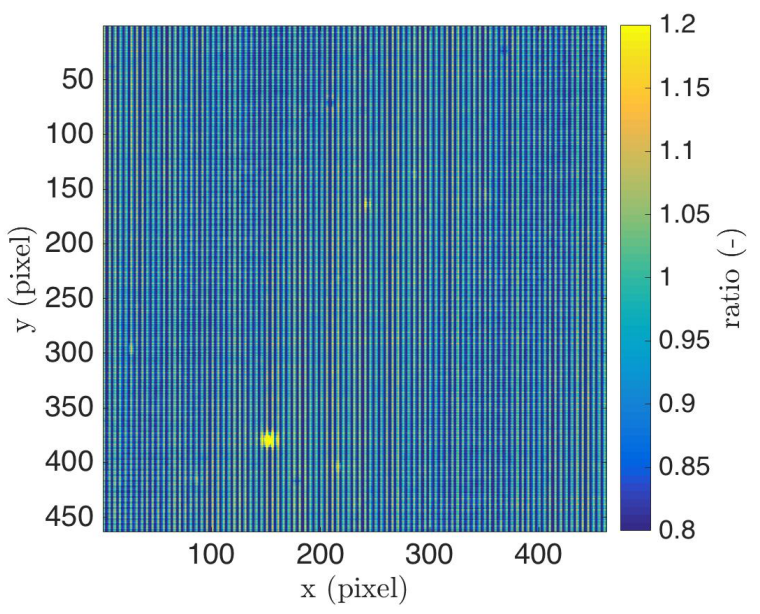

b- $\sigma=5, M=3$

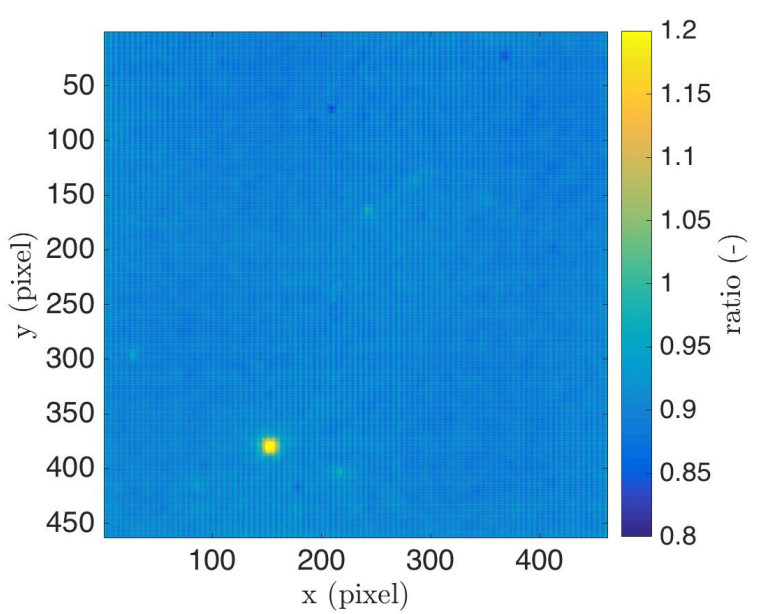

d- average distribution for the nine combinations

Figure 14: a-Grid image under study. The subsets and windows used in the calculations are superimposed. The corresponding spatial resolutions estimated for a given Bias 2 equal to $20 \%$ are plotted as a straight line segment in front of each subset/window. b- to d- examples of ratio between $\widetilde{\alpha}$ calcutated piwelwise for GM and DIC. The same dimensions are used for the four sub-figures. 
with the results obtained with the grid image processed by GM.

\subsubsection{Normalization}

The product between displacement and spatial resolution defined above can be calculated again in each of the five cases. Comparing the metrological performance of DIC and GM from the obtained results would however be biased by some factors which are not really intrinsic to DIC, but which are external to the DIC technique itself. This mainly concerns the displacement resolution, the spatial resolution being theoretically unaffected. The lighting conditions, the gray depth used when recording the pictures and eventually the dynamic range of the image of the pattern are typical examples, these quantities changing between the images used here. A normalization of the results obtained by processing these images is therefore necessary in some way prior to use them for comparison purposes between DIC and GM.

It is proposed here to locally normalize the obtained results by the local gradient of the image, the noise level in final maps being inversely proportional to this gradient for both techniques. It means that $\widetilde{\sigma}_{u}$ must be multiplied by the local image gradient norm denoted above $\bar{\nabla}$ to obtain another normalized displacement resolution denoted $\widetilde{\sigma}_{u}$. By "local gradient", we mean the average value of the norm of the gradient calculated over the subset for DIC. For GM, a convolution between the window used in the WFT and the pixelwise distribution of the gradient norm is performed. Note that this quantity is known as the local total variation in the image processing literature [66]. This procedure gives eventually a pattern-independent normalized displacement resolution denoted $\widetilde{\widetilde{\sigma}}_{u}$. It is defined by

$$
\widetilde{\widetilde{\sigma}}_{u}=\widetilde{\sigma}_{u} \times \bar{\nabla}
$$

The unit of $\widetilde{\sigma}_{u}$ is that of $\widetilde{\sigma}_{u}$ (pixel/DN) multiplied by that of $\bar{\nabla}(\mathrm{DN} /$ pixel), so there is no unit for this quantity. Let us justify more rigorously this type of normalization:

For DIC We consider here the simplest noise propagation model proposed in Equation 24, it can be shown that an upper bound for $\widetilde{\sigma}_{u}$ is inversely proportional to the mean gradient $\bar{\nabla}$ (see also Equation 5-97 in Ref. [31]), which justifies for DIC the normalization introduced in Equation 40.

For GM The following ideal sine profile for the lines of the regular patterns processed by GM is now considered. We consider the 1D case for the sake of simplicity. This gives:

$$
s(x)=\frac{1}{2}\left(1+\gamma A \sin \frac{2 \pi x}{p}\right)
$$

The gradient along $x$ of the line profile is equal to $\frac{\gamma A \pi}{p} \cos \left(\frac{2 \pi x}{p}\right)$, so the mean value $\bar{\nabla}$ of the norm is proportional to $\frac{\gamma A}{p}$. When considering now Equation 35, we can see that with GM, $\widetilde{\sigma}_{u}$ is proportional to $\frac{p}{K}$. In addition and according to [16], $K$ is equal to

$$
K=\frac{\gamma A}{4}
$$

which means that $\widetilde{\sigma}_{u}$ is proportional to $\frac{p}{\gamma A}$, and thus inversely proportional to $\bar{\nabla}$. This conclusion also justifies for GM the normalization proposed in Equation 40. 


\subsubsection{Results and discussion}

We define first from preceding definitions the pattern-independent normalized metrological indicator as

$$
\widetilde{\widetilde{\alpha}}=d_{u} \times \widetilde{\widetilde{\sigma}}_{u}
$$

This quantity enables us to assess the difference in the results obtained by DIC performed on speckles on the one hand, and DIC performed on a grid image on the other hand. The ratio between $\widetilde{\widetilde{\alpha}}$ of two of the five speckles (Speckle 1 and Speckle 2), and $\widetilde{\widetilde{\alpha}}$ of the grid image shown in Figure 13, is considered in practice. Two values for $M$ are considered: $M=6$ and $M=20$. The first one is sufficient for patterns containing small details like the grid pattern. The second one is more suitable for coarser patterns like Speckle 4. Figure 15 shows typical examples of the spatial distribution of this ratio for two speckles as well as the corresponding histograms.

Strong spatial fluctuations are clearly visible because speckles and grid patterns are uncorrelated. On average, the ratio is lower than one. This is clearly visible on the histograms. It means that in this example, performing correlation on speckled patterns leads to a higher noise in displacement maps due to camera sensor noise propagation than when performing correlation on grid images. More generally, we consider now the mean value for this ratio over the maps. Table 2 gathers the results in these ten cases $(5$ speckles $\times 2$ values for $M$ ). Interestingly, the obtained values are lower than one in nine cases, and slightly greater than one in one case. We can see that the lowest values are attained for Speckles 3 and 4, which are the coarsest of the series, and for $2 M+1=13$.

\begin{tabular}{|c|ccccc|}
\hline Subset size $2 M+1$ & Speckle 1 & Speckle 2 & Speckle 3 & Speckle 4 & Speckle 5 \\
\hline 13 & 0.9007 & 0.8554 & 0.7230 & 0.7891 & 0.9430 \\
\hline 41 & 0.9304 & 0.9300 & 0.9117 & 1.0021 & 0.9766 \\
\hline
\end{tabular}

Table 2: Mean of the ratio between $\widetilde{\widetilde{\alpha}}$ calcutated piwelwise by DIC between the grid and the speckle images shown in Figure 13. Subset size $2 M+1: 13$ and 41.

This result shows that the results concerning DIC which are discussed in the preceding sections, and which were obtained with grid images, are globally representative of the more general case of speckle patterns. Speckle patterns are however expected to give results which are less favorable compared to those obtained with grid images.

To compare now results obtained with grid images processed by GM, and speckle or grid images obtained by DIC, we represent in Figure 16 the cumulative distribution function of $\widetilde{\widetilde{\alpha}}$ obtained for the six markings shown in Figure 13. These curves represent the percentage of pixels (along the vertical direction) for which $\widetilde{\widetilde{\alpha}}$ reaches a certain value which is reported along the horizontal direction. We consider here the different settings used in the preceding discussions for DIC and GM: $M=$ $3,6,10,15,20$ pixels and $\sigma=5,10,15$ pixels, respectively, but we only represent the results obtained with the smallest and the highest values of these parameters. Hence the cases $M=3$ pixels for DIC and $\sigma=5$ pixels for GM is represented in Figure 16-a, while $M=20$ pixels for DIC and $\sigma=15$ pixels for GM is represented in Figure 16-b.

The ideal shape for this distribution should satisfy the following properties:

- P1: This curve should be a perfect vertical line to illustrate that $\widetilde{\widetilde{\alpha}}$ remains constant at any pixel of the image;

- P2: This vertical line should be as close as possible to the origin (thus close to the left-hand side of the figure) to illustrate that the best possible compromise between spatial resolution and displacement resolution is achieved. 

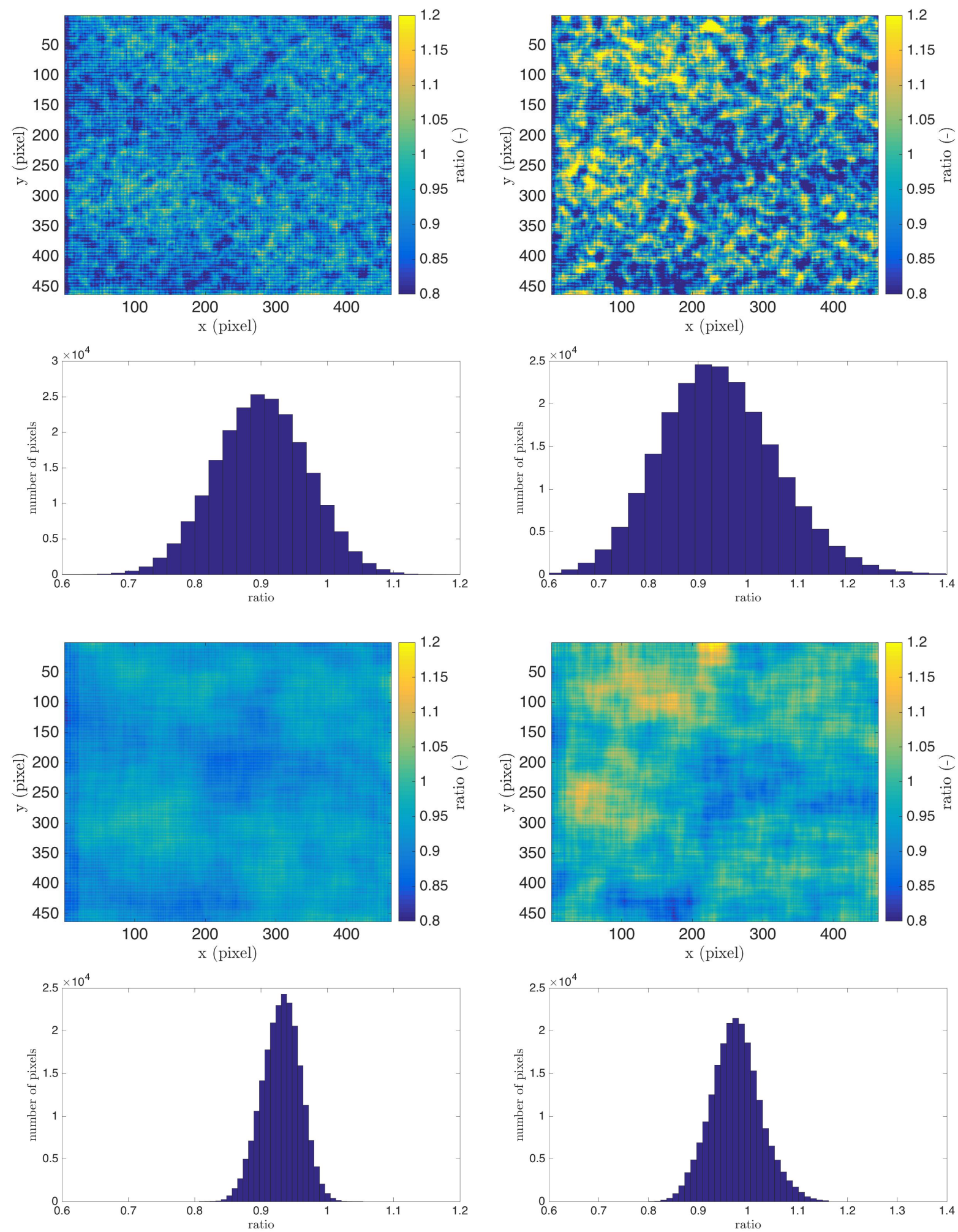

Figure 15: Ratio between $\widetilde{\widetilde{\alpha}}$ calcutated piwelwise for grid image and Speckle 1 (left) and Speckle 5 (right). $M=6$ in the two first lines, $M=20$ in the two last lines. 


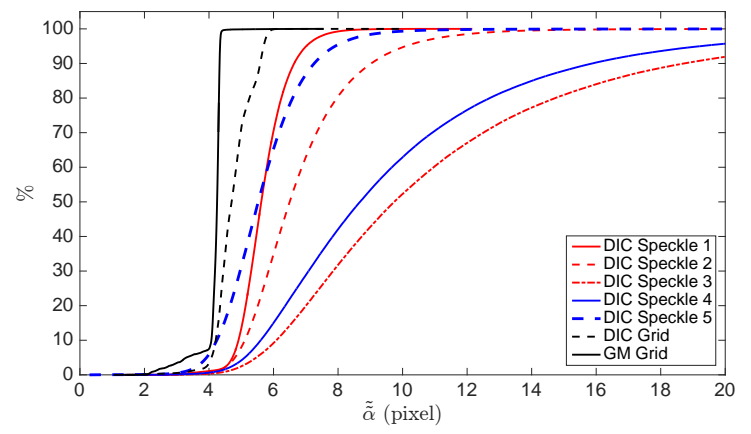

a- DIC: $M=3$ pixels, GM: $\sigma=5$ pixels

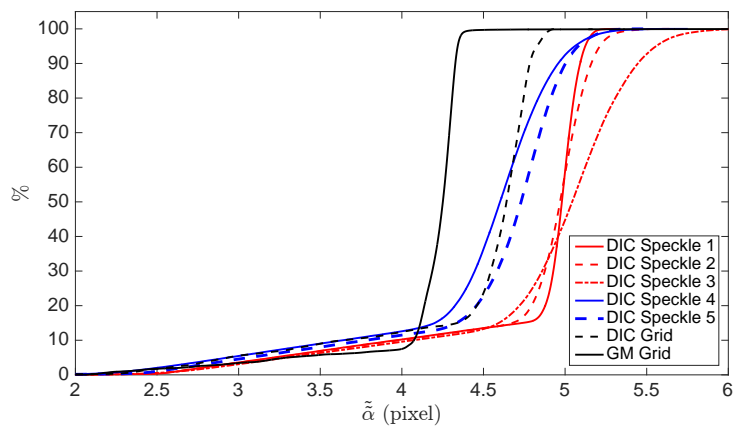

b- DIC: $M=20$ pixels, GM: $\sigma=15$ pixels

Figure 16: Cumulative distribution function determined for various speckle and grid images and settings (without interpolation for DIC). $\widetilde{\widetilde{\alpha}}$ is calculated pixelwise. Neither the effect of Bias 3 nor that of the "spatial noise" are taken into account in these results.

The following remarks can be drawn from the curves shown in Figure 16:

- None of the curves perfectly fulfills both requirements listed above. All of them form an S slanted so that its base and top are horizontal. However, the curve for GM is nearly a vertical line: only the low end of the three curves plotted for this technique, which represents about $10 \%$ of the pixels, exhibits a non-constant value for $\widetilde{\widetilde{\alpha}}$. The remaining $90 \%$ exhibit a nearly constant value, which can be explained by the regularity of the marking;

- Property P1 is not satisfied for DIC for the smallest values of the subset size $2 M+1$ (see Figure 16-a), but this property only reflects the fact that the subset size is not well suited to the speckles under study. Larger subsets lead to curves which become more vertical (see Figure 16-b);

- When correct setting are used for DIC (see Figure 16-b), all the five curves obtained with DIC are on the right-hand side of the curve obtained with GM. It means that $\widetilde{\widetilde{\alpha}}$ is higher for DIC than for GM, thus that the compromise between spatial resolution and displacement resolution is better for GM than for DIC for all these five speckles;

The preceding remark is illustrated by Figure 17, which shows the relative difference between the average abscissa of the vertical portion of the cumulative distribution function of $\widetilde{\alpha}$ calculated for DIC, and its counterpart calculated for GM. The first quantity is estimated for each of the six markings ( 5 speckles and the grid). To avoid the influence of the tails of these distributions, only the points belonging to the 15\%-95\% range along the vertical direction of each of these cumulative distribution functions are considered in this calculation. Depending on the pattern, $\widetilde{\widetilde{\alpha}}$ is between 10 and $20 \%$ greater for speckles processed by DIC than for grids processed by GM, which means that the compromise between displacement resolution and spatial resolution is better for GM than for DIC in these examples. It is worth remembering here that this results holds for DIC performed pixelwise. Interpolation between remote points would further impair the results found for DIC, as illustrated in Section 3.3. Taking into account the heteroscedasticity of the noise in real images would also certainly amplify this difference in response, the grid image being much darker than the speckles shown in Figure 13.

\section{Conclusion}

A comparative study between DIC and GM is presented in this paper. Because of the lack of precise standard available on the literature on the assessment of the metrological performance of full-field 


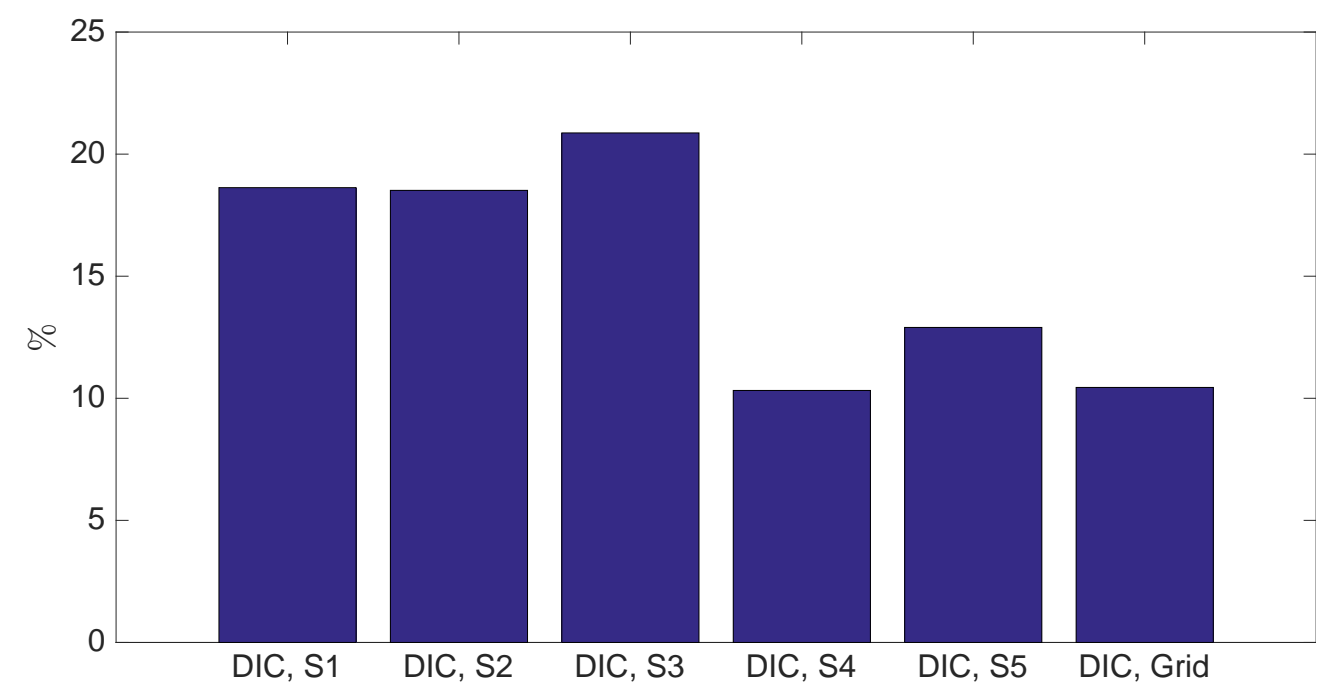

Figure 17: Relative difference between DIC and GM (without interpolation for DIC).

measurement systems, a suitable methodology had to be proposed to perform a fair comparison. The metrological performance of both techniques was assessed in terms of bias, displacement resolution and spatial resolution. Special attention was paid to characterize and compare quantities considered as intrinsic to the techniques such as the link between these three quantities when they are available. The size of the subset for DIC or the size of the analysis window for GM are not really metrological quantities of interest. These quantities are settings fixed by the user, so they were more considered as technical parameters, which are specific to each technique. Another striking point is that grid images were processed by DIC to obtain results with both techniques on the same set of images, although this procedure is not conventional for DIC. Speckle images were also processed and the obtained results were compared to those obtained with GM after applying a suitable normalization. As a general remark, these first results show that GM globally exhibits a better compromise between displacement resolution and spatial resolution for a given attenuation bias (Bias 2). Interpolation between remote points, which is classically used in DIC, has the most significant negative impact on the metrological performance as defined here.

The study presented here is not completely exhaustive. The first reason is the lack of information available in the literature on some couplings such as that which certainly exists between Bias 1 and the spatial resolution. The second reason is that the "spatial noise" has not been taken into account to assess the displacement resolution. This is due to the fact that there is a lack of models able to reliably predict this phenomenon. This justifies that additional studies should be undertaken to tackle this issue correctly for both techniques. The third reason is that other criteria such as the computational cost or the ease with which the surface of the specimen is prepared, should be taken into account in a more global comparison. The computational cost depends on the way the methods are programmed. It may therefore strongly change from one version of the same technique to another. On this point, GM relies on image processing tools based on Fourier analysis, which have been studied in depth in the computer science community for decades. Concerning surface preparation, it is clear that depositing a grid for GM is trickier than spraying speckles on uniformly painted surfaces for DIC. The first type of marking is however reproducible, which can be an advantage for standardization purposes. As a general remark, it can be said that GM and DIC are more complementary than competing. From this first comparison between both techniques and even though the obtained results are partial, it turns out that GM should be used in situations for which low strain levels, and high gradients occur, while DIC is certainly the best solution in the other cases, the additional effort necessary to deposit regular 
grids instead of spraying speckles being then not justified. Depending on the parameter potential users attach importance to, the limit between these two types of situations may fluctuate, and thus the final choice for using one of the two techniques for a given application may be different.

As a last remark, it is worth recalling that we relied here on predictive formulas. Even though they were validated in earlier studies, readers interested in verifying these results experimentally should bear in mind that external parameters may strongly affect the results obtained with such experiments. Out-of-plane motion occurring during the tests or micro-vibrations between specimen and camera are typical examples.

In conclusion, though incomplete since other effects, criteria or parameter settings could also be accounted for, this first attempt helps to critically compare subset-based local DIC and GM in an

objective and fair manner, and the methodology proposed here could also be used in other cases, for instance to compare different versions of the same technique.

\section{Acknowledgement}

The GDR CNRS ISIS is gratefully acknowledged for its partial financial support of this study (TIMEX project). 


\section{Appendix 1: formal link between DIC and GM}

The aim of this calculation is to give the formal link that exists between DIC and GM. Let $s^{\text {ref }}$ and $s^{\text {cur }}$ be two images taken before and after deformation, respectively. In local DIC, estimating the $2 \mathrm{D}$ displacement $\left(u_{x}, u_{y}\right)$ at a given point $\left(x_{0}, y_{0}\right)$ consists in minimizing the squared optical residual in a neighbourhood $\Omega$ of $\left(x_{0}, y_{0}\right)$, which writes:

$$
\iint_{(x, y) \in \Omega}\left(s^{r e f}(x, y)-s^{c u r}\left(x+u_{x}, y+u_{y}\right)\right)^{2} \mathrm{~d} x \mathrm{~d} y
$$

where $\left(x_{0}, y_{0}\right) \in \Omega$. This is a continuous version of Equation 1 used with DIC. By a slight abuse of notation, we keep on denoting by $s^{r e f}$ and $s^{\text {cur }}$ the restriction of both images $s^{r e f}$ and $s^{c u r}$ to $\Omega$, this restriction being extended to $\mathbb{R}^{2}$ as $s^{r e f}(x, y)=0$ and $s^{\text {cur }}(x, y)=0$ when $(x, y)$ is not in $\Omega$. Thus Equation 44 writes:

$$
\iint_{(x, y) \in \mathbb{R}^{2}}\left(s^{r e f}(x, y)-s^{c u r}\left(x+u_{x}, y+u_{y}\right)\right)^{2} \mathrm{~d} x \mathrm{~d} y
$$

Extending to $\mathbb{R}^{2}$ the domain of integration enables us to use the Parseval theorem (also named Plancherel theorem), which states that, under mild assumptions, any function $f$ and its Fourier transform $\widehat{f}$ are such that $\int_{\mathbb{R}^{2}}|f|^{2}=K \int_{\mathbb{R}^{2}}|\widehat{f}|^{2}$, where $K$ is some constant independent from $f$, and $|z|$ is the norm of any complex number $z$. In addition, denoting $s^{\text {cur }}(x, y)=s^{c u r}\left(x+u_{x}, y+u_{y}\right)$ and assuming that the displacement $\left(u_{x}, u_{y}\right)$ is constant over $\Omega$, we have $\widehat{s^{\prime c u r}}(\xi, \eta)=e^{2 i \pi\left(\xi u_{x}+\eta u_{y}\right)} \widehat{s^{c u r}}(\xi, \eta)$. As a consequence, minimizing the squared optical residual $\iint\left(s^{r e f}-s^{\text {Icur }}\right)^{2}$ (cf. Equation 45 ) is equivalent to minimizing

$$
\iint_{(\xi, \eta) \in \mathbb{R}^{2}}\left|\widehat{s^{r e f}}(\xi, \eta)-e^{2 i \pi\left(\xi u_{x}+\eta u_{y}\right)} \widehat{s^{c u r}}(\xi, \eta)\right|^{2} \mathrm{~d} \xi \mathrm{d} \eta
$$

For any complex number $z_{1}$ and $z_{2},\left|z_{1}-z_{2}\right|^{2}=\left|z_{1}\right|^{2}+\left|z_{2}\right|^{2}-2 \operatorname{Re}\left(z_{2} \overline{z_{1}}\right)$ where $\operatorname{Re}(z)$ denotes the real part and $\bar{z}$ the complex conjugate. The quantity defined in Equation 46 is thus equal to:

$$
\iint_{(\xi, \eta) \in \mathbb{R}^{2}}\left(\left|\widehat{s^{r e f}}(\xi, \eta)\right|^{2}+\left|\widehat{s^{c u r}}(\xi, \eta)\right|^{2}-2 \operatorname{Re}\left(e^{2 i \pi\left(\xi u_{x}+\eta u_{y}\right)} \widehat{s^{c u r}}(\xi, \eta) \widehat{\widehat{s^{r e f}}}(\xi, \eta)\right)\right) \mathrm{d} \xi \mathrm{d} \eta
$$

The optimum translation $\left(u_{x}, u_{y}\right)$ has to satisfy Euler-Lagrange equation, that is, the derivative of the integrand with respect to $u_{x}$ and $u_{y}$ must be null. As a consequence, for any $\xi, \eta$,

$$
\left\{\begin{array}{l}
\operatorname{Re}\left(2 i \pi \xi e^{2 i \pi\left(\xi u_{x}+\eta u_{y}\right)} \widehat{\widehat{s^{c u r}}}(\xi, \eta) \widehat{\widehat{s^{r e f}}}(\xi, \eta)\right)=0 \\
\operatorname{Re}\left(2 i \pi \eta e^{2 i \pi\left(\xi u_{x}+\eta u_{y}\right)} \widehat{\widehat{s^{c u r}}}(\xi, \eta) \widehat{\widehat{s^{r e f}}}(\xi, \eta)\right)=0
\end{array}\right.
$$

Both equalities eventually give:

$$
\arg \left(e^{2 i \pi\left(\xi u_{x}+\eta u_{y}\right)} \widehat{s^{c u r}}(\xi, \eta) \widehat{\widehat{s^{r e f}}}(\xi, \eta)\right)=0[\pi]
$$

where arg is the argument of any non-zero complex number, the equation holding modulo $\pi$. Since we are looking for a minimum, the second derivative of the integrand must be positive, thus

$$
\left\{\begin{array}{l}
-2 \operatorname{Re}\left(-4 \pi^{2} \xi^{2} e^{2 i \pi\left(\xi u_{x}+\eta u_{y}\right)} \widehat{s^{\text {cur }}}(\xi, \eta) \widehat{\widehat{s^{r e f}}}(\xi, \eta)\right)>0 \\
-2 \operatorname{Re}\left(-4 \pi^{2} \eta^{2} e^{2 i \pi\left(\xi u_{x}+\eta u_{y}\right)} \widehat{s^{\text {cur }}}(\xi, \eta) \widehat{\widehat{s^{r e f}}}(\xi, \eta)\right)>0
\end{array}\right.
$$


A consequence if that Equation 49 actually holds modulo $2 \pi$ instead of $\pi$. Indeed, for any $\xi$ and $\eta, e^{2 i \pi(\xi u+\eta v)} \widehat{s^{c u r}}(\xi, \eta) \widehat{s^{r e f}}(\xi, \eta)$ is a real number from Equation 49 , which must be positive from Equation 50. Consequently:

$$
\arg \left(e^{2 i \pi\left(\xi u_{x}+\eta u_{y}\right)} \widehat{s^{c u r}}(\xi, \eta) \widehat{\widehat{s^{r e f}}}(\xi, \eta)\right)=0[2 \pi]
$$

We can conclude that, for any $(\xi, \eta)$, the displacement $\left(u_{x}, u_{y}\right)$ satisfies:

$$
\xi u_{x}+\eta u_{y}=-\frac{1}{2 \pi} \arg \left(\widehat{s^{c u r}}(\xi, \eta) \widehat{\widehat{s^{r e f}}}(\xi, \eta)\right)
$$

This calculation is not particular to grid images; it is valid for any pair of translated images. Retrieving $u_{x}$ and $u_{y}$ from Equation 52 is the basis of the so-called phase correlation method [67]. Two approaches are recalled in [67] to solve this problem. With the first one, $u_{x}$ and $u_{y}$ can be estimated through a multivariate linear regression on the scatter plot of $(\xi, \eta)$ against the values of $\arg \left(\widehat{s_{2}}(\xi, \eta) \widehat{\widehat{s^{r e f}}}(\xi, \eta)\right) /(2 \pi)$ computed from the discrete Fourier transform of both images $s^{\text {ref }}$ and $s^{\text {cur }}$. With the second method, since Equation 52 is equivalent to:

$$
A e^{2 i \pi\left(\xi u_{x}+\eta u_{y}\right)}=\widehat{s^{c u r}}(\xi, \eta) \widehat{\widehat{s^{r e f}}}(\xi, \eta)
$$

where $A>0$, the inverse Fourier transform of the right-hand member of this equality should be a Dirac distribution located at $\left(u_{x}, u_{y}\right)$. As a consequence, finding $\left(u_{x}, u_{y}\right)$ consists in localizing the corresponding peak. While the first approach suffers from phase wrapping and noise, the second one is not adapted to cases where $u, v$ are below one pixel, or needs a careful interpolation to reach such low values [68].

As far as images of grids of nominal pitch $p$ are concerned, it is possible to further simplify Equation 52. The spectrum of grid images has been thoroughly studied in [45] by using the stationary phase method. It has been shown that this spectrum is made of separated spikes lying around the harmonics of the grid, the spikes being precisely expressed by Equation 9 of [45] (adapted to match the notations of the present paper). For instance, for any $(\xi, \eta)$ around the fundamental harmonics $\left(\frac{1}{p}, 0\right)$, the following equation holds:

$$
\xi u_{x}+\eta u_{y}=-\frac{1}{2 \pi} \arg \left(\widehat{e^{i \Phi_{x}^{\text {cur }}}}\left(\xi-\frac{1}{p}, \eta\right) \widehat{\overline{e^{i \Phi_{x}^{r e f}}}}\left(\xi-\frac{1}{p}, \eta\right)\right)
$$

and for any $(\xi, \eta)$ around the fundamental harmonics $\left(0, \frac{1}{p}\right)$ :

$$
\xi u_{x}+\eta u_{y}=-\frac{1}{2 \pi} \arg \left(\widehat{e^{i \Phi_{y}^{\text {cur }}}}\left(\xi, \eta-\frac{1}{p}\right) \widehat{e^{i \Phi_{y}^{\text {ref }}}}\left(\xi, \eta-\frac{1}{p}\right)\right)
$$

$u_{x}$ and $u_{y}$ can be retrieved from these equations by using several approaches, for instance the Geometric Phase Analysis (GPA) [41], its windowed version [43], or the localized spectrum analysis [17] used in the present study.

If the phase modulations $\Phi_{x}$ and $\Phi_{y}$ can be considered as constant in $\Omega$ (that is, no phase modulation caused by grid manufacturing constraints is allowed), then $\widehat{e^{i \Phi}}=e^{i \Phi} \delta_{(0,0)}$ where $\delta$ is the Dirac distribution. In this case, Equations 54-55 give:

$$
u_{i}=-\frac{p}{2 \pi}\left(\Phi_{i}^{c u r}-\Phi_{i}^{r e f}\right) \quad i \in\{x, y\}
$$


which is Equation 11 used with GM.

As a conclusion, it can be said that under mild assumption, minimizing the optical residual on a subset requires iterative calculations in case of random pattern (DIC), but is straightforward in case of regular pattern (GM). It means that assuming that the displacement field and the phase modulations are constant within the subset, both approaches should theoretically give the same estimation. This demonstration argues that both methods should theoretically provide the same solution if the small strain assumptions holds, since in this case displacement fields are nearly constant. In practice, both methods differ because of several reasons. It is possible to mention: algorithmic considerations (how to set the stopping criteria?), practical considerations (grids are not perfectly periodic and speckles show areas where pixel intensity does not provide any information, the gradient being nul for instance), noise propagation, which differs in both methods. Since no unifying theoretical framework exists yet to compare both methods with respect to these criteria, this motivates the numerical experiments provided to the reader in the present paper. 


\section{Appendix 2: Savitzky-Golay coefficients}

In the case of DIC, for a matching function of degree $d$ and for a subset size $2 M+1$, the $h(i)=h_{M, d}^{D I C}(i)$ coefficients involved in Equation 20, $i=0 \cdots M$ are defined by:

$$
h_{M, d}^{D I C}(i)=\frac{p_{i}}{n o r m}
$$

The values for the $p_{i}$ coefficients and for norm are defined in Tables 3 to 5 in the case $d=2$.

\begin{tabular}{|cccc|c|}
\hline$p_{0}$ & $p_{1}$ & $p_{2}$ & $p_{3}$ & norm \\
\hline 7 & 6 & 3 & -2 & 21 \\
\hline
\end{tabular}

Table 3: Savitzky-Golay coefficients, $d=2, M=3[56]$.

\begin{tabular}{|ccccccc|c|}
\hline$p_{0}$ & $p_{1}$ & $p_{2}$ & $p_{3}$ & $p_{4}$ & $p_{5}$ & $p_{6}$ & norm \\
\hline 25 & 24 & 21 & 16 & 9 & 0 & -11 & 143 \\
\hline
\end{tabular}

Table 4: Savitzky-Golay coefficients, $d=2, M=6[56]$.

\begin{tabular}{|ccccccccccc|c|}
\hline$p_{0}$ & $p_{1}$ & $p_{2}$ & $p_{3}$ & $p_{4}$ & $p_{5}$ & $p_{6}$ & $p_{7}$ & $p_{8}$ & $p_{9}$ & $p_{10}$ & norm \\
\hline 329 & 324 & 309 & 284 & 249 & 204 & 149 & 84 & 9 & -76 & -171 & 3059 \\
\hline
\end{tabular}

Table 5: Savitzky-Golay coefficients, $d=2, M=10$ [56]. 


\section{References}

[1] Z. Y. Wang, H. Q. Li, J. W. Tonga, and J. T. Ruan. Statistical analysis of the effect of intensity pattern noise on the displacement measurement precision of digital image correlation using selfcorrelated images. Experimental Mechanics, 47(5):701-707, 2007.

[2] M. Bornert, F. Brémand, P. Doumalin, J.-C. Dupré, M. Fazzini, M. Grédiac, F. Hild, S. Mistou, J. Molimard, J.-J. Orteu, L. Robert, Y. Surrel, P. Vacher, and B. Wattrisse. Assessment of digital image correlation measurement errors: methodology and results. Experimental Mechanics, 49(3):353-370, 2009.

[3] H. Haddadi and S. Belhabib. Use of a rigid-body motion for the investigation and estimation of the measurement errors related to digital image correlation technique. Optics and Lasers in Engineering, 46(2):185-96, 2008.

[4] P. Lava, S. Cooreman, S. Coppieters, M. DeStrycker, and D. Debruyne. Assessment of measuring errors in DIC using deformation fields generated by plastic FEA. Optics and Lasers in Engineering, 47(7):747-753, 2009.

[5] J. Réthoré. A fully integrated noise robust strategy for the identification of constitutive laws from digital images. Internation Journal for Numerical Methods in Engineering, 84(6):631-660, 2010.

[6] P. Reu. Calibration: A good calibration image. Experimental Techniques, 37(6):1-3, 2013.

[7] P. Reu. Speckles and their relationship to the digital camera. Experimental Techniques, 38(4):1-2, 2014.

[8] M. Badaloni, M. Rossi, G. Chiappini, P. Lava, and D. Debruyne. Impact of experimental uncertainties on the identification of mechanical material properties using DIC. Experimental Mechanics, 55(8):1411-1426, 2015.

[9] P. Reu. All about speckles: Contrast. Experimental Techniques, 39(1):1-2, 2015.

[10] R. Gras, H. Leclerc, F. Hild, S. Roux, and J. Schneider. Identification of a set of macroscopic elastic parameters in a $3 \mathrm{~d}$ woven composite: Uncertainty analysis and regularization. International Journal of Solids and Structures,, 55:2-16, 2015.

[11] L. Wittevrongel, P. Lava, S. V. Lomov, and D. Debruyne. A self adaptive global digital image correlation algorithm. Experimental Mechanics, 55(2):361-378, 2015.

[12] Y. Wang, P. Lava, P. Reu, and D. Debruyne. Theoretical analysis on the measurement errors of local 2D DIC: Part I part i temporal and spatial uncertainty quantification of displacement measurements. Strain, 52(2):110-128, 2016.

[13] B. Blaysat, M. Grédiac, and F. Sur. Effect of interpolation on noise propagation from images to DIC displacement maps. International Journal for Numerical Methods in Engineering, 108(3):213-232, 2016.

[14] B. Blaysat, M. Grédiac, and F. Sur. On the propagation of camera sensor noise to displacement maps obtained by DIC. Experimental Mechanics, 56(6):919-944, 2016.

[15] F. Sur and M. Grédiac. Towards deconvolution to enhance the grid method for in-plane strain measurement. Inverse Problems and Imaging, 8(1):259-291, 2014. American Institute of Mathematical Sciences. 
[16] M. Grédiac and F. Sur. Effect of sensor noise on the resolution and spatial resolution of the displacement and strain maps obtained with the grid method. Strain, 50(1):1-27, 2014. Paper invited for the 50th anniversary of the journal.

[17] M. Grédiac, F. Sur, and B. Blaysat. The grid method for in-plane displacement and strain measurement: a review and analysis. Strain, 52(3):205-243, 2016.

[18] F. Sur and M. Grédiac. Influence of the analysis window on the metrological performance of the grid method. Journal of Mathematical Imaging and Vision, 56(3):472-498, 2016.

[19] E. A. Patterson, E. Hack, P. Brailly, R. L. Burguete, Q. Saleeme, T. Siebert, R. A. Tomlinsone, and M. P. Whelan. Calibration and evaluation of optical systems for full-field strain measurement. Optics and Lasers in Engineering, 45(5):550-564, 2007.

[20] E. Hack, G. Lampeas, J.E. Mottershead, E.A. Patterson, T. Siebert, and M.P. Whelan. Progress in developing a standard for dynamic strain analysis. In Experimental and Applied Mechanics, volume 6 of Conference Proceedings of the Society for Experimental Mechanics Series, pages 425-429, 2011.

[21] C. Sebastian, X. Lin, E. Hack, and E. Patterson. A reference material for establishing uncertainty for static and dynamic displacements. In Proceedings of the SEM conference, Costa Mesa, CA, USA, 2015. to appear.

[22] E. Hack, X. Lin, E.A. Patterson, and C.M. Sebastian. A reference material for establishing uncertainties in full-field displacement measurements. Measurement Science and Technology, 26(7):075004, 2015.

[23] http://cordis.europa.eu/result/brief/rcn/3262_en.html.

[24] http://www.dynamicvalidation.org/.

[25] http://www.engineeringvalidation.org/.

[26] Standard guide for evaluating non-contacting optical strain measurement systems, ASTM standard E2208-02, 2010.

[27] JCGM 200. International vocabulary of metrology. basic and general concepts and associated terms, 2008.

[28] http://www.emva.org/wp-content/uploads/EMVA1288-3.1rc2.pdf.

[29] P. Doumalin, M. Bornert, and D. Caldemaison. Microextensometry by image correlation applied to micromechanical studies using the scanning electron microscopy. In Japanese Society for Experimental Mechanics, editor, Proceedings of the international conference on advanced technology in experimental mechanics, pages 81-86, 1999. Ube City, Japan.

[30] H. W. Schreier and M. A. Sutton. Systematic errors in digital image correlation due to undermatched subset shape functions. Experimental Mechanics, 42(3):303-310, 2002.

[31] M. Sutton, J.J. Orteu, and H. Schreier. Image Correlation for Shape, Motion and Deformation Measurements. Basic Concepts, Theory and Applications. Springer, 2009.

[32] R. Fedele, L. Galantucci, and A. Ciani. Global 2d digital image correlation for motion estimation in a finite element framework: a variational formulation and a regularized, pyramidal, multi-grid implementation. International Journal for Numerical Methods in Engineering, 96(12):739-762, 2013. 
[33] G. Besnard, F. Hild, and S. Roux. Finite-element displacement fields analysis from digital images: application to Portevin-Le Chatelier bands. Experimental Mechanics, 46(6):1-15, 2006.

[34] Y. Sun, J. H. L. Pang, C. K. Wong, and F. Su. Finite element formulation for a digital image correlation method. Applied Optics, 44(34):7357-7363, 2005.

[35] F. Hild and S. Roux. Comparison of local and global approaches to digital image correlation. Experimental Mechanics, 52(9):1503-1519, 2012.

[36] B. Pan, B. Wang, G. Lubineau, and A. Moussawi. Comparison of subset-based local and finite element-based global digital image correlation. Experimental Mechanics, 55(5):887-901, 2015.

[37] B. Wang and B. Pan. Subset-based local vs. finite element-based global digital image correlation: A comparison study. Theoretical and Applied Mechanics Letters, 6(5):200-208, 2016.

[38] J. Neggers, B. Blaysat, J.P.M. Hoefnagels, and M.G.D. Geers. On image gradients in digital image correlation. International Journal for Numerical Methods in Engineering, 105(4):243-260, 2016.

[39] C. Badulescu, M. Grédiac, and J.-D. Mathias. Investigation of the grid method for accurate in-plane strain measurement. Measurement Science and Technology, 20(9):20:095102, doi:10.1088/0957-0233/20/9/095102, 2009. IOP.

[40] S. Ri, M. Fujigaki, and Y. Morimoto. Sampling moiré method for accurate small deformation distribution measurement. Experimental Mechanics, 50(4):501-508, 2010.

[41] M. J. Hÿtch, E. Snoeck, and R. Kilaas. Quantitative measurement of displacement and strain fields from HREM micrographs. Ultramicroscopy, 74:131-146, 1998.

[42] X. Dai, H. Xie, H. Wang, C. Li, Z. Liu, and L. Wu. The geometric phase analysis method based on the local high resolution discrete fourier transform for deformation measurement. Measurement Science and Technology, 25(2):025402, 2014.

[43] X. Dai, H. Xie, and H. Wang. Geometric phase analysis based on the windowed fourier transform for the deformation field measurement. Optics and Laser Technology, 58(6):119-127, 2014.

[44] Y. Surrel. Photomechanics, Topics in Applied Physic, volume 77, chapter Fringe Analysis, pages 55-102. Springer, 2000.

[45] F. Sur, B. Blaysat, and M. Grédiac. Determining displacement and strain maps immune from aliasing effect with the grid method. Optics and Lasers in Engineering, 86:317-328, 2016.

[46] J. M. Huntley. Noise-immune phase unwrapping algorithm. Applied Optics, 28(16):3268-3270, 1989.

[47] M.A. Arevallilo Herraez, J.G. Boticario, M.J. Lalor, and D.R. Burton. Agglomerative clusteringbased approach for two-dimensional phase unwrapping. Applied optics, 41(35):7437-7444, 2002.

[48] http://www.thegridmethod.net.

[49] ISO 5725. Accuracy (trueness and precision) of measurement methods and results, 1994. the International Organization for Standardization.

[50] H. Schreier, W. Hubert, J. R. Braasch, and M. Sutton. Systematic errors in digital image correlation caused by intensity interpolation. Optical Engineering, 39(11):2915-2921, 2000. 
[51] P. L. Reu. Experimental and numerical methods for exact subpixel shifting. Experimental Mechanics, 51(4):443-452, 2011.

[52] Y. Su, Q. Zhang, Z. Gao, X. Xu, and X. Wu. Fourier-based interpolation bias prediction in digital image correlation. Optics Express, 23(15):19242-19260, 2015.

[53] W. Tong. Formulation of Lucas-Kanade digital image correlation algorithms for non-contact deformation measurements: a review. Strain, 49(4):313-334, 2013.

[54] C. Badulescu, M. Grédiac, J.-D. Mathias, and D. Roux. A procedure for accurate one-dimensional strain measurement using the grid method. Experimental Mechanics, 49(6):841-854, 2009.

[55] Y. Q. Wang, M.Sutton, H. Bruck, and H. W. Schreier. Quantitative error assessment in pattern matching: Effects of intensity pattern noise, interpolation, strain and image contrast on motion measurements. Strain, 45(2):160 -178, 2009.

[56] A. Savitzky and M.J.E. Golay. Smoothing and differentiation of data by simplified least-squares procedures. Analytical Chemistry, 36(3):1627-1639, 1964.

[57] E. W. Grafarend. Linear and Nonlinear Models: Fixed Effects, Random Effects, and Mixed Models. Walter de Gruyter, 2006.

[58] M. Grédiac, F. Sur, C. Badulescu, and J.-D. Mathias. Using deconvolution to improve the metrological performance of the grid method. Optics and Lasers in Engineering, 51(6):716-734, 2013.

[59] L. Yu and B. Pan. The errors in digital image correlation due to overmatched shape functions. Measurement Science and Technology, 26(4):045202, 2015.

[60] C. Badulescu, M. Grédiac, H. Haddadi, J.-D. Mathias, X. Balandraud, and H.-S. Tran. Applying the grid method and infrared thermography to investigate plastic deformation in aluminium multicrystal. Mechanics of Materials, 43(11):36-53, 2011.

[61] A. Chrysochoos and Y. Surrel. Chapter 1. Basics of metrology and introduction to techniques. In M. Grédiac and F. Hild, editors, Full-field measurements and identification in solid mechanics, pages 1-29. Wiley, 2012.

[62] B. Zhao and Y. Surrel. Effect of quantization error on the computed phase of phase-shifting measurements. Applied Optics, 36(12):2070-2075, 1997.

[63] A. Foi, M. Trimeche, V. Katkovnik, and K. Egiazarian. Practical Poissonian-Gaussian noise modeling and fitting for single-image raw-data. IEEE Transactions on Image Processing, 17(10):1737$1754,2008$.

[64] B. Pan, H. Xie, Z. Wang, K. Qian, and Z. Wang. Study on subset size selection in digital image correlation for speckle patterns. Optics Express, 16(10):7037-7048, 2008.

[65] C. Badulescu, M. Bornert, J.-C. Dupré, S. Equis, M. Grédiac, J. Molimard, P. Picart, R. Rotinat, and V. Valle. Demodulation of spatial carrier images: Performance analysis of several algorithms. Experimental Mechanics, 53(8):1357-1370, 2013.

[66] A. Buades, T. M. Le, J. M. Morel, and L. A. Vese. Fast cartoon + texture image filters. IEEE Transactions on Image Processing, 19(8):1978-1986, 2010.

[67] H. Foroosh, J.B. Zerubia, and M. Berthod. Extension of phase correlation to subpixel registration. IEEE Transactions on Image Processing, 11(3):188-200, 2002. 
[68] D. J. Chen, F. P. Chiang, Y. S. Tan, and H. S. Don. Digital speckle-displacement measurement using a complex spectrum method. Applied Optics, 32(11):1839-1849, 1993. 